\title{
Eruptive xanthoma model reveals endothelial cells internalize and metabolize chylomicrons, leading to extravascular triglyceride accumulation
}

\author{
Ainara G. Cabodevilla, ${ }^{1}$ Songtao Tang, ${ }^{1}$ Sungwoon Lee, ${ }^{2}$ Adam E. Mullick, ${ }^{3}$ Jose O. Aleman, ${ }^{1}$ M. Mahmood Hussain, ${ }^{4}$ \\ William C. Sessa, ${ }^{2}$ Nada A. Abumrad, ${ }^{5}$ and Ira J. Coldberg ${ }^{1}$ \\ 'Division of Endocrinology, Diabetes and Metabolism, Department of Medicine, New York University Grossman School of Medicine, New York, New York, USA. ${ }^{2}$ Vascular Biology and Therapeutics Program, \\ Yale University School of Medicine, New Haven, Connecticut, USA. ${ }^{3}$ Ionis Pharmaceuticals, Carlsbad, California, USA. ${ }^{4}$ Diabetes and Obesity Center, NYU-Long Island School of Medicine, Mineola, New York, \\ USA. ${ }^{5}$ Nutritional Sciences, Department of Medicine and Department of Cell Biology and Physiology, Washington University School of Medicine, Saint Louis, Missouri, USA.
}

\begin{abstract}
Although tissue uptake of fatty acids from chylomicrons is primarily via lipoprotein lipase (LpL) hydrolysis of triglycerides (TGs), studies of patients with genetic LpL deficiency suggest additional pathways deliver dietary lipids to tissues. Despite an intact endothelial cell (EC) barrier, hyperchylomicronemic patients accumulate chylomicron-derived lipids within skin macrophages, leading to the clinical finding eruptive xanthomas. We explored whether an LpL-independent pathway exists for transfer of circulating lipids across the EC barrier. We found that LpL-deficient mice had a marked increase in aortic EC lipid droplets before and after a fat gavage. Cultured ECs internalized chylomicrons, which were hydrolyzed within lysosomes. The products of this hydrolysis fueled lipid droplet biogenesis in ECs and triggered lipid accumulation in cocultured macrophages. EC chylomicron uptake was inhibited by competition with HDL and knockdown of the scavenger receptor-BI (SR-BI). In vivo, SR-BI knockdown reduced TC accumulation in aortic ECs and skin macrophages of LpL-deficient mice. Thus, ECs internalize chylomicrons, metabolize them in lysosomes, and either store or release their lipids. This latter process may allow accumulation of TGs within skin macrophages and illustrates a pathway that might be responsible for creation of eruptive xanthomas.
\end{abstract}

\section{Introduction}

The delivery of dietary lipids for metabolic use in peripheral tissues is a fundamental biological process in all vertebrates. In mammals, these lipids circulate mostly as components of lipoproteins in the form of phospholipids (PLs), triglycerides (TGs), and cholesteryl esters (CEs). For tissues without fenestrated capillaries, lipid uptake requires the passage of lipoproteins or their components across the endothelial barrier (1). However, despite the importance of this process in whole-body metabolism and homeostasis, how lipids cross the endothelium is still not fully understood.

Due to their relevance in the physiopathology of atherosclerosis, endothelial uptake and transcytosis of cholesterolcarrying lipoproteins LDL and HDL have been extensively studied, and a series of receptors have recently been implicated in this process (reviewed in ref. 2). In contrast, most lipid delivery from larger TG-rich lipoproteins (TRLs) VLDL and chylomicrons does not appear to involve transcytosis and uptake of whole lipoproteins. The canonical pathway for delivery of TRL-derived lipids to parenchymal tissues involves lipolysis at

Authorship note: AGC and ST are co-first authors

Conflict of interest: AEM is an employee of Ionis Pharmaceuticals.

Copyright: (5) 2021, American Society for Clinical Investigation.

Submitted: November 11, 2020; Accepted: April 28, 2021; Published: June 15, 2021.

Reference information: J Clin Invest. 2021;131(12):e145800.

https://doi.org/10.1172/JCl145800. the capillary surface by lipoprotein lipase (LpL) and, at low fatty acid (FA) concentrations, FA transporter proteins, such as cluster of differentiation 36 (CD36) (3-6). LpL is synthesized in the parenchyma of metabolically active tissues, such as heart, skeletal muscle, and adipose $(7,8)$, and is anchored to endothelial cells (ECs) at the luminal surface of the capillary via its interaction with glycosylphosphatidylinositol-anchored HDL-binding protein 1 (GPIHBP1) (9). There, LpL mediates the lipolysis of TGs, allowing released FAs to be taken up into parenchymal cells. However, clinical and experimental evidence indicate that additional pathways independent of LpL must mediate the uptake of TRL-derived lipids.

Patients with genetic loss of LpL, its obligate binding GPIHBP1, its coactivator apoC2, or lipase maturation factor 1 (LMF1) exhibit defective TRL lipolysis, fasting hyperchylomicronemia, and recurrent pancreatitis (10-13). Fasting hyperchylomicronemia is a rare disorder, and its relationship to cardiovascular disease (CVD) is unclear (14-16). Also unclear is why these patients, who have a major defect in lipid metabolism, do not exhibit defective adipose development or impaired heart or skeletal muscle mechanics. In contrast with patients who have defective production of chylomicrons due to defects in either apoB or microsomal TG transfer protein (17), LpL-deficient patients do not exhibit visual, neurological, immunological, or coagulation defects due to fat-soluble vitamin deficiency.

LpL-deficient patients develop cutaneous eruptive xanthomas, papular lesions characterized by the accumulation of lipid- 
laden macrophages in the skin (18). The biological process underlying this pathological sign remains poorly understood. Eruptive xanthomas are overlaid by an intact endothelium, which would preclude the paracellular passage of unhydrolyzed TLRs. However, lipids within xanthoma skin macrophages are strikingly similar to those carried by circulating chylomicrons (19), suggesting that chylomicron lipids cross the endothelial barrier in the absence of LpL-mediated lipolysis. Together, these clinical observations suggest that a non-LpL pathway for delivery of dietary lipids must be operative. In support of this hypothesis, Kuo et al. reported that mouse aortic ECs accumulate lipid droplets (LDs) in the postprandial period, when levels of circulating chylomicrons are elevated (20). In WT mice, these LDs could have been generated from lipolysis-derived FAs, followed by intracellular reassembly of TG, internalization of nonlipolyzed chylomicrons, or a combination of these 2 events. Aortic ECs are not surrounded by high LpL-expressing cells, and very few cells express GPIHBP1 $(21,22)$. Therefore, LpL lipolysis products are unlikely to be the primary substrate for aortic endothelial LD biogenesis.

Here, we provide evidence that postprandial LD accumulation in aortic ECs is independent of LpL activity and requires EC uptake of unhydrolyzed chylomicrons via scavenger receptor-BI (SR-BI), followed by chylomicron hydrolysis within lysosomes. To illustrate this LpL-independent pathway, we assessed TG accumulation in skin macrophages, a model for the development of eruptive xanthomas. We show that conditioned medium from chylomicron-treated ECs induces LD biogenesis in cocultured macrophages and that knockdown of SR-BI decreases TG accumulation within skin macrophages of hyperchylomicronemic mice. Our results imply that uptake of chylomicrons by ECs may be an important and underappreciated pathway for the delivery of circulating TRL-derived lipids that is exclusive of intravascular lipolysis.

\section{Results}

Postprandial aortic LDs are not lipolysis derived. To determine whether postprandial LD biogenesis within aortic ECs requires LpL lipolysis, we monitored LD occurrence over time (270 minutes) following olive oil gavage in control and inducible LpL-knockout (iL $p \mathrm{l}^{-/}$) mice (Figure 1A). Aortas harvested at the indicated times were immunostained for endothelial-specific membrane protein VE-cadherin (red), and LDs were stained with neutral lipid dye 4,4-difluoro-1,3,5,7,8-pentamethyl-4bora-3a,4a-diaza-s-indacene (BODIPY 493/503) (green) for confocal microscopy visualization and analysis. Manufacturer and catalogue number information for reagents used in this study can be found in Supplemental Table 1; supplemental material available online with this article; https://doi.org/10.1172/JCI145800DS1. Immunostaining for LD coat protein perilipin 2 is shown in Supplemental Figure 1. Aortas from i $\mathrm{pl}^{-/-}$mice accumulated more LDs within aortic ECs. As opposed what occurred in control mice, LDs were found prior to the gavage (Figure 1A). As expected, LpL deficiency resulted in significantly elevated postprandial TG levels (Figure 1B). With increased TG levels, the number of LDs within aortic ECs markedly increased, so that by 270 minutes, $\mathrm{i} L \mathrm{pl}^{-/}$mice had approximately 4 fold more LDs than $L p^{f l / f l}$ controls (Figure 1, $A$ and B; quantified in Figure 1C).
We have previously shown that mice with endothelial-specific knockout of CD36 exhibit impaired uptake of long-chain FAs into heart, skeletal muscle, and brown adipose tissue as well as elevated postprandial TG levels (4). To assess whether CD36-mediated endothelial FA uptake is required for postprandial LD biogenesis in aortic ECs, we administered an olive oil gavage to $\mathrm{EC}-\mathrm{Cd} 36^{-/-}$ and EC-Cd36 $6^{f / f l}$ mice and monitored plasma TG and aortic EC LD content over time. As in LpL-deficient mice, both postprandial TG and LD content within aortic ECs were increased in EC-Cd36 mice as compared with floxed controls (Figure 1D). Plasma TG levels and LDs per cell are shown in Figure 1, E and F.

Next, we assessed LD formation in cultured ECs exposed to purified TRLs. Immediately following a fat load, TGs circulate mostly as components of chylomicrons (23). Therefore, mouse ECs (MECs) were deprived of serum overnight to ensure minimal LD content and then exposed to purified human chylomicrons (4mg/ dL TG) in FBS-free medium for 120 minutes. Cells were immunostained for LD coat protein perilipin 2 in addition to BODIPY 493/503. MECs deprived of serum overnight exhibited only background signal of both perilipin 2 (red) and BODIPY 493/503 (green), indicating LD depletion (Figure 1G). In the absence of LpL or any other lipid source, MECs exposed to chylomicrons formed LDs, assessed by the presence of perilipin 2/BODIPY 493/503positive puncta (Figure $1 \mathrm{H}$ ). Pretreatment with $10 \mathrm{U} / \mathrm{mL} \mathrm{LpL}$ with or without heparin enhanced accumulation of LDs (Figure 1, I and $\mathrm{J}$ ), which were significantly larger than those arising from incubation with chylomicrons alone (quantified in Figure $1 \mathrm{~K}$ ). In this setting, LD size strongly correlated with the concentration of FAs in the media after treatment, which was increased in cells pretreated with LpL (Figure 1, L and M).

These observations suggest that, although LpL-hydrolyzed chylomicron FAs may contribute to postprandial LD biogenesis in ECs, tissues with little or no LpL expression are likely to have other lipid-uptake mechanisms.

ECs exposed to purified chylomicrons exhibit 2 populations of $T G$-rich droplets. To further characterize the nature of postprandial endothelial LDs, we exposed aortas from WT mice to purified human chylomicrons ex vivo. LDs were visible within ECs of VE-cadherin- and BODIPY 493/503-stained aortas after a 30- or 120-minute exposure to chylomicrons (Figure 2A). Particle-size analysis of intracellular BODIPY 493/503-positive structures evidenced that endothelial LDs were significantly larger at longer incubations, and similar results were obtained in cultured MECs exposed to chylomicrons in vitro (Figure 2B). In both settings, LD size more than doubled at 120-minute relative to 30-minute incubations (Figure 2C).

MECs exposed to chylomicrons for 120 minutes exhibited large (average size, 250 nm) BODIPY 493/503-positive droplets (green) which colocalized with perilipin 2 (red) (Figure 2, D and E). However, there was no detectable perilipin 2 signal or colocalization with small (average size, $100 \mathrm{~nm}$ ) BODIPY 493/503-positive puncta at short (30 minutes) incubation times (Figure 2, D and E).

Each chylomicron carries 1 molecule of apoB48, the amino terminal $48 \%$ of apoB100, which is produced from the $A P O B$ gene in the intestine by an mRNA-editing process (24). To assess whether the smaller ( $100 \mathrm{~nm}$, Figure 2D) BODIPY 493/503-positive structures represented internalized chylomicrons, we exposed 
A
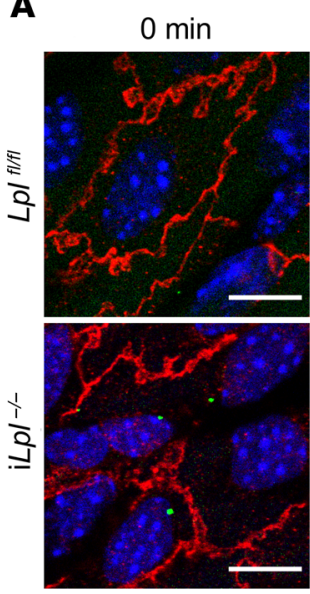

B

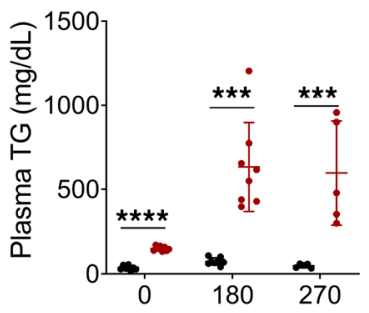

$180 \mathrm{~min}$

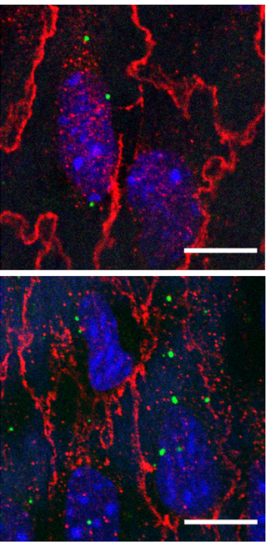

C

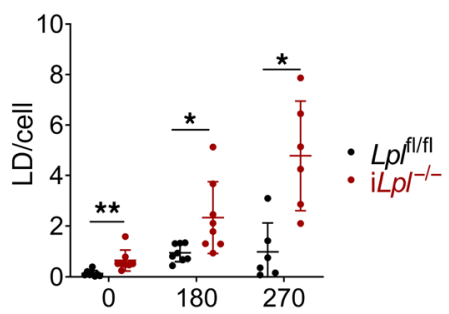

D

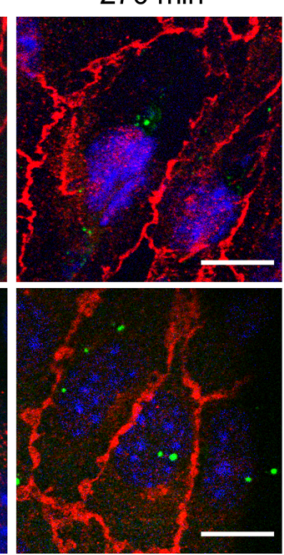

$0 \min$
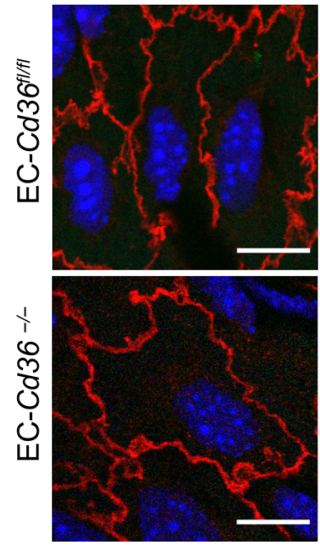

E

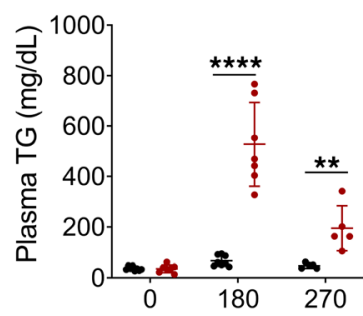

$180 \mathrm{~min}$
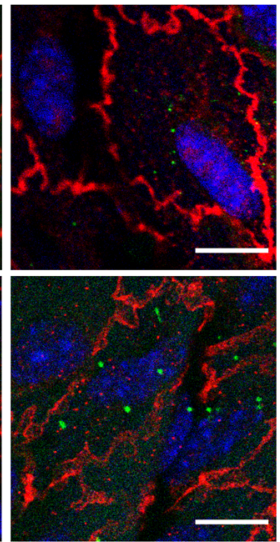

$\mathbf{F}$

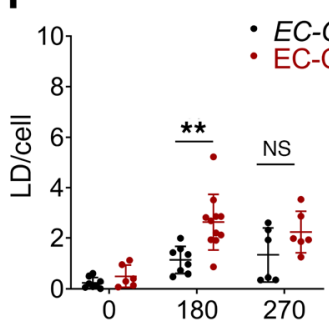

G

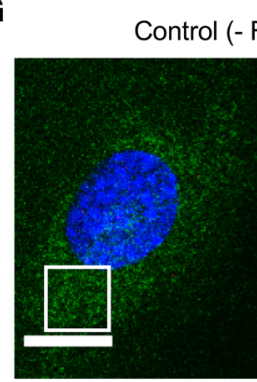

K

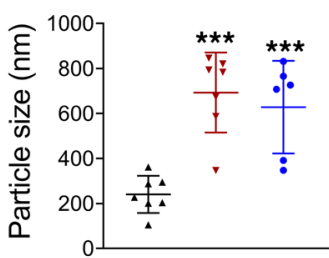

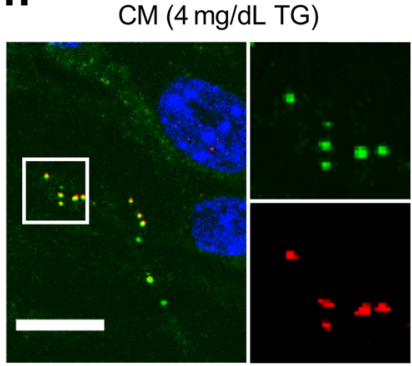

I

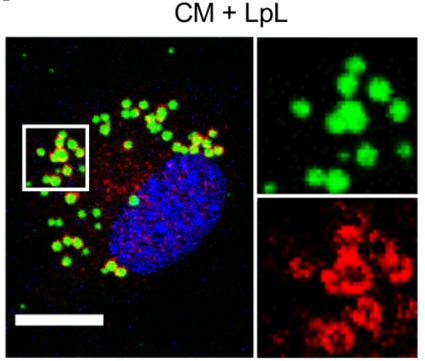

$270 \min$

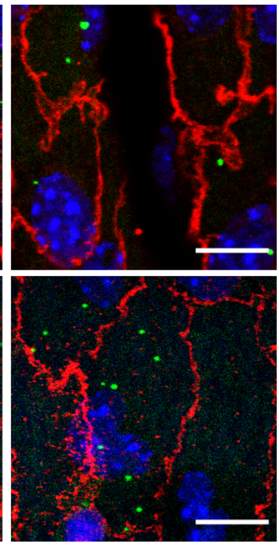

EC-Cd36 f/ffl $\mathrm{EC}-\mathrm{Cd} 36^{-1-}$

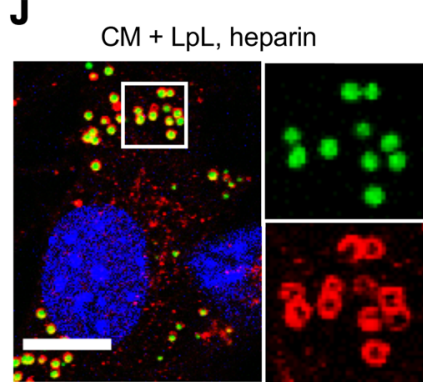

$\mathbf{L}$

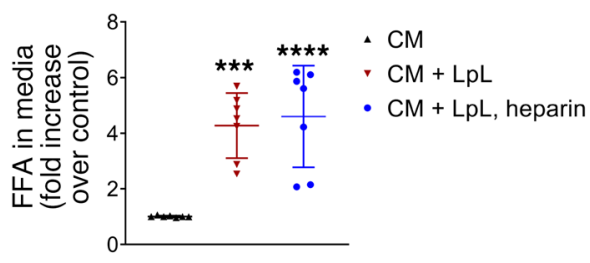

M

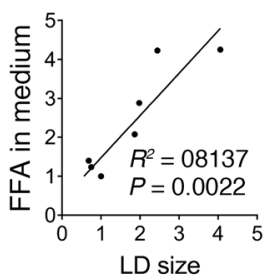

Figure 1. Mouse aortic ECs accumulate LDs after an olive oil gavage in the absence of $L p L$ or endothelial CD36. Three- to four-month-old male $\left.i L p\right|^{f /|f|}$,

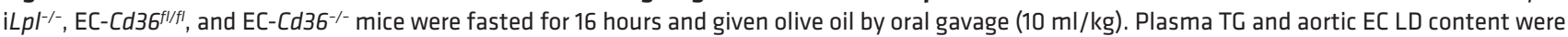
assessed at the indicated times ( $n=5-8$ mice/group/time point). (A-C) Both iLp/fl/fl and iLpl-/- mice exhibited BODIPY 493/503-positive (green) LDs within aortic ECs, as visualized by confocal microscopy imaging of $\mathrm{V}$-cadherin-immunostained (red) samples (A). Unlike floxed controls, iLp/ ${ }^{-/-}$mice exhibited LDs within aortic ECs prior to the olive oil gavage (A, left panels). In the absence of LpL lipolysis, plasma TC (B) and LD accumulation (C) were significantly increased. (D-F) EC-specific CD36 deletion resulted in an increase in aortic EC LD content (D, quantified in F), which was mirrored by elevated plasma TC levels (E). All comparisons are with flox/flox controls. ${ }^{*} P<0.05$; ${ }^{* *} P<0.01 ;{ }^{* *} P<0.001$; ${ }^{* * * *} P<0.0001$, 2-way ANOVA. (G-M) MECs were deprived of serum overnight to ensure LD depletion $(\mathbf{C})$ and pretreated with $\mathrm{LpL}(10 \mathrm{U} / \mathrm{ml})(\mathbf{I})$ or $\mathrm{LpL}^{+}$heparin $(10 \mathrm{U} / \mathrm{ml})$. (J) or maintained in FBS-free medium (H) for 1 hour before a 120-minute incubation with human chylomicrons. Samples were immunostained with LD marker perilipin 2 (red) and stained with BODIPY 493/503 (green). LDs were significantly larger in the presence of $\mathrm{LpL}^{+/}$heparin pretreatment (K), and their size correlated with the presence of FFAs in the media after treatment $(\mathbf{M})$, which was significantly increased in samples pretreated with $\mathrm{LpL}^{+/-}$heparin $(\mathbf{L})$. Data are represented as mean $\pm \mathrm{SEM}$ of 7 independent experiments. ${ }^{* *} P<0.01$; ${ }^{* *} P<0.001 ;{ }^{* * *} P<0.0001,1$-way ANOVA, Dunnet's multiple comparisons test. Scale bars: $10 \mu \mathrm{m}$. Additional inset magnification, $\times 2.5$. 
cultured MECs to purified chylomicrons for 30 or 120 minutes and immunostained for apoB. Similar levels of intracellular apoBpositive puncta were detected following 30- or 120-minute incubations with chylomicrons (Figure $2 \mathrm{~F}$ ). At 30 minutes, apoB fully colocalized with BODIPY 493/503-positive puncta (Figure $2 \mathrm{G}$ ). However, colocalization at 120 minutes was partial, observed only in the population of smaller BODIPY 493/503-positive structures (Figure 2H). These data suggest that at 120-minute incubation, newly biosynthesized LDs coexist with internalized chylomicrons within ECs. Chylomicrons obtained from i $L \mathrm{pl}^{t^{-}}$mice, as well as from an LpL-deficient patient, were also tested and yielded similar results (Supplemental Figure 2, A and B).

Intracellular apoB signal disappears over time. ECs exposed to chylomicrons in culture formed LDs in the absence of LpL lipolysis or any other lipid source. In addition, intracellular apoBpositive/BODIPY 493/503 puncta were visible early with chylomicron exposure while apoB-negative/perilipin 2-positive/ BODIPY 493/503 LDs only appeared at longer incubation times. These observations suggested that internalized chylomicrons provide the substrate for LD biogenesis in ECs. To assess this, we first monitored apoB and BODIPY 493/503 signals over time in MEC pulsed with human chylomicrons for 30 minutes and then switched to FBS-free culture medium for 120 minutes. Approximately $80 \%$ of MEC exhibited approximately $100 \mathrm{~nm}$ intracellular apoB/ BODIPY 493/503-positive puncta after a pulse with chylomicrons (Figure 3A). The apoB signal rapidly declined when MECs were switched to FBS-free medium (Figure 3B and quantification in Figure 3C; time course in Supplemental Figure 3A), but the number of cells still exhibiting cytoplasmic BODIPY 493/503positive puncta did not change (Figure 3D). However, the LD at 120 minutes were significantly larger $(200 \mathrm{~nm}$; Figure $3 \mathrm{E})$ and did not colocalize with apoB (as shown in Figure 3B).

$L D$ biogenesis in ECs requires chylomicron hydrolysis in lysosomes. De novo LD biogenesis in eukaryotic cells occurs in the endoplasmic reticulum, typically in response to excess intracellular FAs (25-28). To determine whether intracellular chylomicron hydrolysis was the source of FAs for LD biogenesis in MECs, we studied the effect of 2 specific inhibitors on intracellular chylomicron clearance and LD appearance. Atglistatin is a selective inhibitor of adipose TG lipase (ATGL), the rate-limiting enzyme involved in the mobilization of FAs from intracellular TG stores (29-32). Bafilomycin A1 (BafA1), commonly used to inhibit lysosomal degradation, selectively inhibits the vacuolar-type $\mathrm{H}^{+}$-ATPase (V-ATPase) (33), preventing acidification of organelles and activation of lysosome lipase $(34,35)$. As expected, MECs pulsed with chylomicrons for 30 minutes exhibited intracellular apoB/ BODIPY 493/503 puncta (white merge of magenta and green) (Figure 3F), and apoB staining was lost when cells were switched to FBS-free medium for 120 minutes (Figure 3G). Inhibition of ATGL did not block degradation of intracellular chylomicrons, as monitored by loss of cytoplasmic apoB/BODIPY 493/503 puncta, nor appearance of large LDs 120 minutes after a chylomicron pulse (Figure $3 \mathrm{H}$ ). Conversely, inhibition of the lysosomal proton pump with BafA1 resulted in the retention of apoB/ BODIPY 493/503-positive puncta throughout the experiment (Figure 3I). Importantly, MECs treated with BafA1 did not develop large LDs. Although ATGL inhibition did not block chylomicron degradation or LD biogenesis, it resulted in a significant increase of LD size (Figure 3J), in keeping with reports that ATGL is required for LD mobilization in ECs (20).

Endocytosis inhibitor dynasore blocks EC chylomicron uptake. Several receptors are involved in the recognition, binding, and uptake of chylomicron remnants (CMRs) by the liver. LDL receptor (LDLR) and LDLR-related protein/ $\alpha 2$-macroglobulin receptor (LRP) recognize CMRs in an apoE-dependent manner following lipolysis by LpL $(36,37)$. SR-BI, as well as heparan sulfate proteoglycans, may be involved in the "capture step" that precedes the LDLR and LRP-mediated endocytosis of CMR in hepatocytes (38). Additionally, macrophages in culture have been reported to internalize and degrade chylomicrons in an apoC-II and macrophage derived LpL-dependent manner (39). However, no receptor has been reported to participate in the uptake of unhydrolyzed chylomicrons. As a first approach to assess the endothelial chylomicron uptake route, we pulsed serum-deprived MECs with chylomicrons in the absence or presence of dynasore to inhibit endocytic uptake $(40,41)$. Treatment with dynasore blocked chylomicron internalization, leading to their accumulation at the plasma membrane of MECs (Figure 4A).

We next aimed to identify the site of chylomicron membrane binding. Binding of CMRs to heparan sulfate proteoglycans in hepatocyte membranes mediates 1 route of uptake $(42,43)$, a process that is abrogated by heparin (44). Treatment with heparin (5 $\mathrm{U} / \mathrm{mL}$ ) had no effect on MEC chylomicron uptake in culture (Supplemental Figure 4), suggesting that binding of chylomicrons to the surface of MECs could be directly through a receptor.

Endothelial lipase (EL) has been shown to provide an alternative pathway for the uptake of lipoprotein-derived FAs into the adipose tissue of LpL-deficient mice (45) as well as to participate in the binding and cellular uptake of LDL and HDL particles (46). The lack of effect of heparin treatment, which releases EL from the EC surface (45), suggested that EL was not involved in the uptake of chylomicrons. To further confirm this, we used siRNA to specifically knock down EL. As shown in Supplemental Figure 5 , knockdown of EL had no effect on the uptake of fluorescencelabeled (labeled with 1,1'-dioctadecyl-3,3,3',3'-tetramethylindocarbocyanine perchlorate [DiI]) chylomicrons (shown in red).

EC chylomicron uptake is independent of LDLR and ALK1. The process of endothelial chylomicron uptake and degradation outlined so far is reminiscent of the LDLR-mediated endocytosis and lysosomal delivery of LDL. The apoB100 in LDL particles is recognized by LDLR, promoting LDL uptake into the cells. The internalized particles are routed to endosomes, where LDL dissociates from LDLR and is transferred to lysosomes for further degradation. Inhibition of the lysosomes with chloroquine (47) or with BafA1 (48) blocks LDL degradation. Further, LDL uptake in cultured cells is suppressed by inhibiting endocytosis with $80 \mu \mathrm{M}$ dynasore $(49,50)$. Chylomicrons lack the LDLR binding sequence of apoB, which is located in the C-terminal half of the full-length protein. However, LDLR also exhibits high affinity for apoE, which is present in both chylomicrons and their remnants. To assess whether LDLR was required for chylomicron uptake in ECs, we administered DiI-labeled chylomicrons (shown in red) retroorbitally to $\mathrm{Ldlr}^{--}$mice and WT controls. Plasma TG was monitored 15 minutes after injection, and thoracic aortas were harvested to 
A
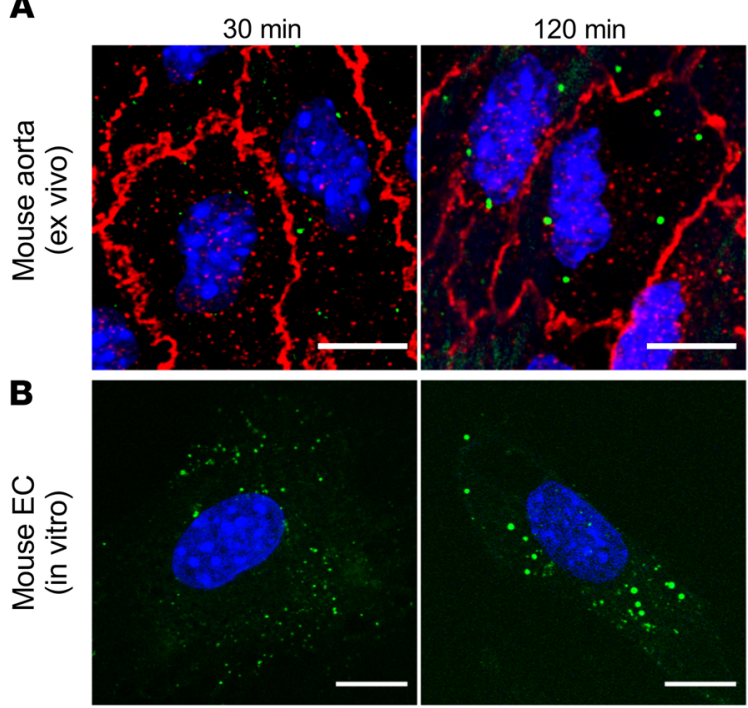

C

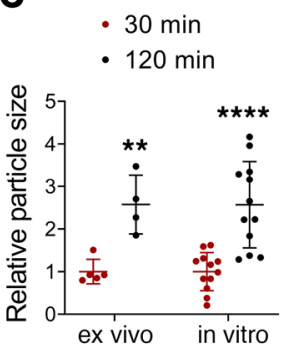

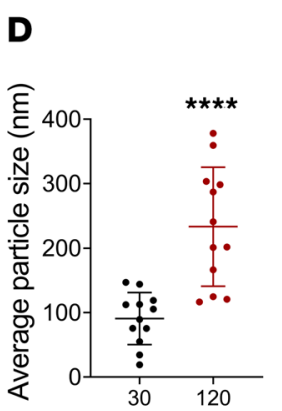

Incubation time ( $\mathrm{min})$

\section{E}
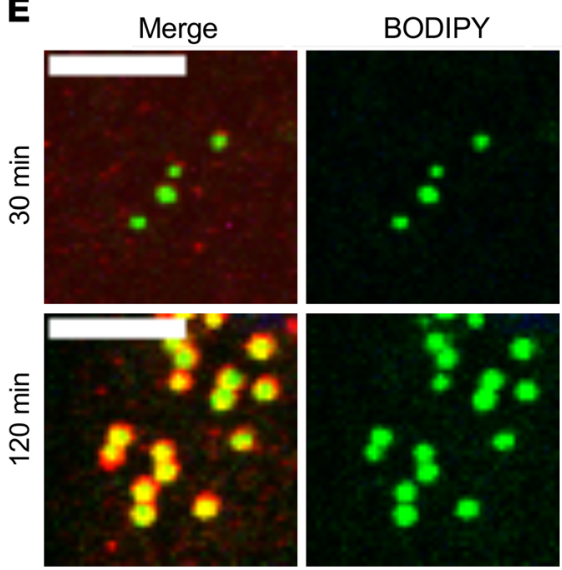

G

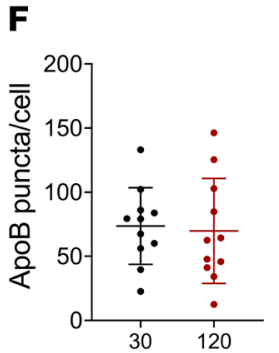

Incubation time $(\min )$

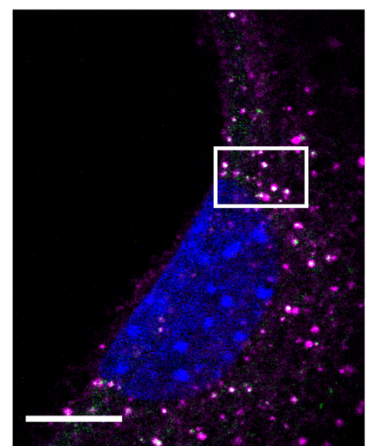

$30 \mathrm{~min}$

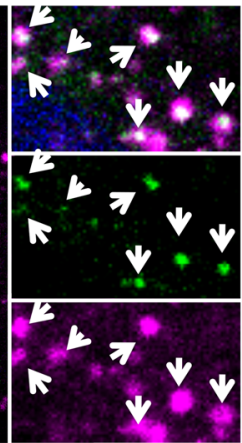

Perilipin 2

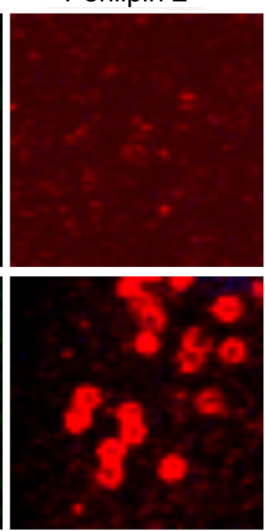

H

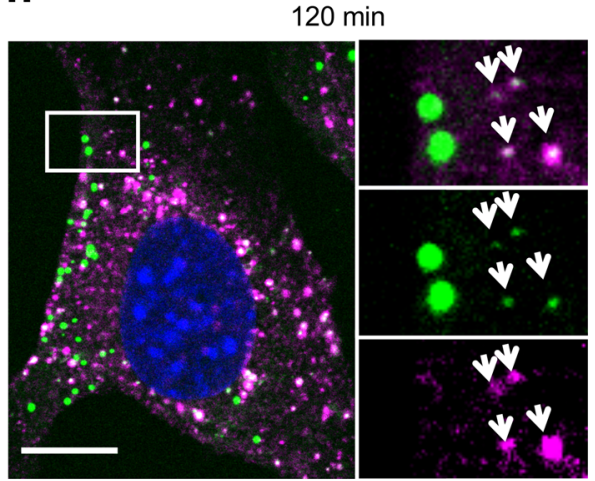

Figure 2. EC BODIPY493/503-positive droplets become larger over time. (A-E) Aortas obtained from fasted WT mice (A) or serum-deprived cultured MECs (B) were incubated with human chylomicrons (4 mg/dL TC in FBS-free medium) for 30 or 120 minutes. Analysis of BODIPY 493/503-positive puncta size (>1000 particles/group) showed a significant increase in particle size over time, with average size increasing more than 2-fold both ex vivo and in vitro (C). Data are represented as mean \pm SEM of 4 (ex vivo) or 12 (in vitro) independent experiments. ${ }^{* *} P<0.01$; ${ }^{* * * *} P<0.0001$, Student's $t$ test. (D and E) MECs were deprived of serum overnight to ensure LD depletion, then incubated with human chylomicrons (4 mg/dL TG) for the indicated times. (D) Average size of BODIPY 493/503-positive puncta after 30 minutes $(\sim 100 \mathrm{~nm})$ or 120 minutes $(\sim 250 \mathrm{~nm})$ exposure to chylomicrons. $n=12$ independent experiments. ${ }^{* * *} P<0.0001$, Student's $t$ test. (E) Immunostaining for perilipin 2 (red). At 120 minutes, BODIPY 493/503-positive droplets (green) colocalized with perilipin 2 (lower panels, colocalization in yellow). There was no perilipin 2 signal or colocalization with BODIPY 493/503-positive droplets at shorter (30 minutes) incubations (upper panels). Scale bars: $5 \mu \mathrm{m}$. (F-H) Immunostaining for lipoprotein marker apoB. MECs incubated with chylomicrons for 30 or 120 minutes exhibited similar numbers of intracellular apoB-positive puncta (F). At 30-minute incubation, BODIPY 493/503-positive puncta (green) fully colocalized with apoB (magenta, C). Colocalization at 120 minutes was partial, with only smaller BODIPY 493/503-positive puncta colocalizing with apoB (H). White arrows indicate apoB/BODIPY 493/503-positive puncta. Scale bars: $10 \mu \mathrm{m}$. Additional inset magnification, $\times 2.5$. 
A

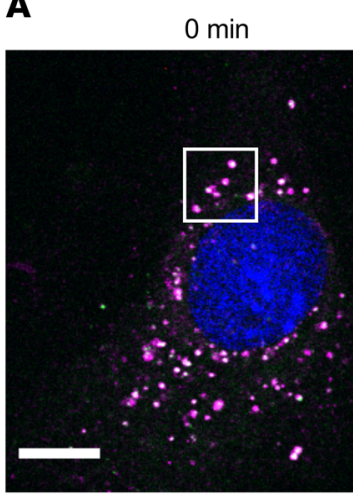

C

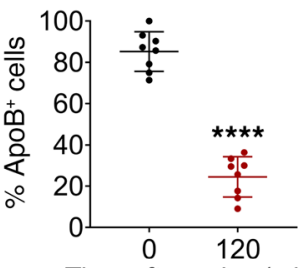

Time after pulse (min)

$\mathbf{F}$

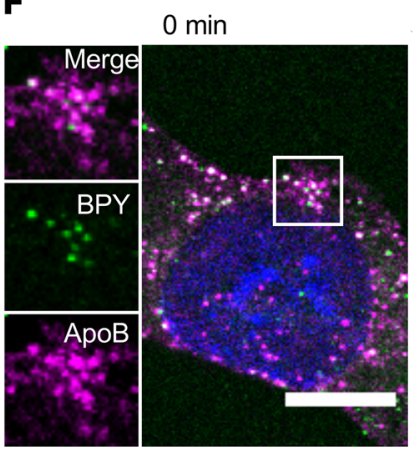

B

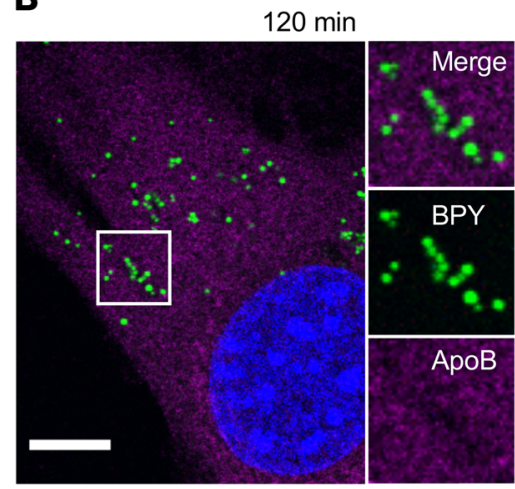

\section{D}

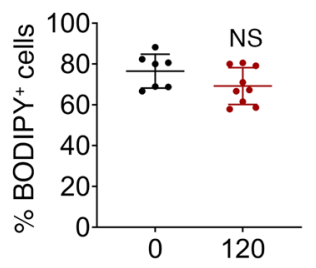

Time after pulse (min)

G

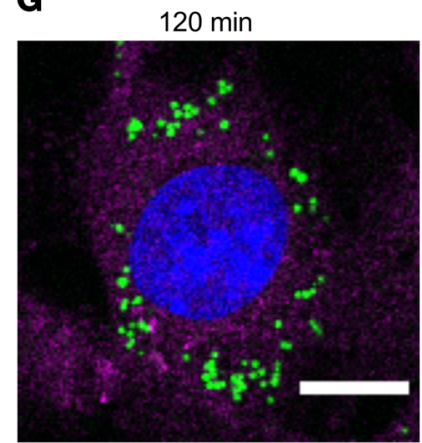

E

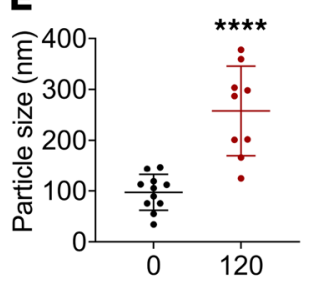

Time after pulse (min)

H

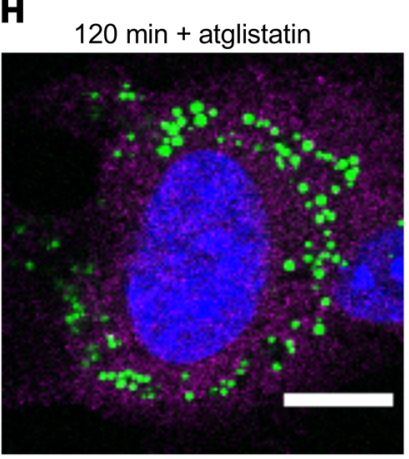

I

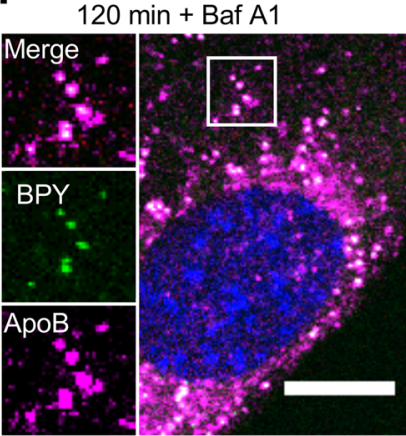

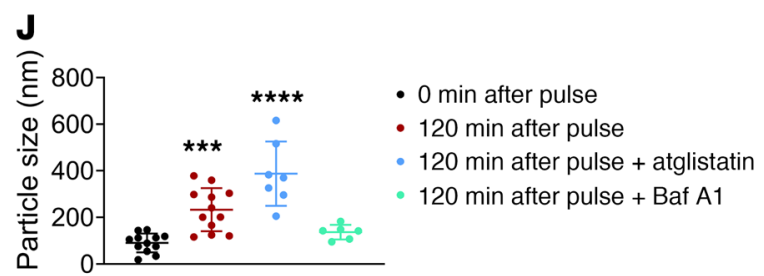

Figure 3. Intracellular chylomicron hydrolysis at the lysosomal compartment precedes LD biogenesis in ECs. (A-E) Cultured MECs were deprived of serum overnight and exposed to human chylomicrons (4 mg/dL in FBS-free medium). After a 30-minute pulse, cells were either fixed ( 0 minutes) or maintained in FBS-free medium for 120 minutes. (A) As expected, cells fixed immediately after the chylomicron pulse exhibited intracellular apoB/ BODIPY 493/503-positive puncta (average size, $100 \mathrm{~nm}$, E). (B) Incubation for 120 minutes in FBS-free medium after pulse resulted in loss of intracellular apoB signal (quantified in C), accompanied by the appearance of larger, BODIPY 493/503-positive puncta (average size $300 \mathrm{~nm}$, E). Data are represented as mean \pm SEM of 8 independent experiments. ${ }^{* * *} P<0.0001$, Student's $t$ test. (F-I) MECs were pulsed for 30 minutes with human chylomicrons, then fixed (F) or switched for 120 minutes to FBS-free medium alone (G) or in the presence of ATGL inhibitor atglistatin (H) or lysosomal proton pump inhibitor BafA1. (I). Average BODIPY 493/503-positive particle size ( $n=7$ independent experiments) is represented in J. Inhibition of ATCL did not preclude chylomicron degradation as monitored by loss of intracellular apoB signal nor the appearance of large ( 300 nm) LDs. Conversely, inhibition of lysosomal hydrolysis with BafA1 resulted in the retention of apoB/BODIPY 493/503-positivecytoplasmic puncta ( 100 nm) for the duration of the treatment. Data are represented as mean $\pm \mathrm{SD} .{ }^{* *} P<0.001 ;{ }^{* * *} P<0.0001,1$-way ANOVA, Dunnet's multiple comparisons test. Scale bars: $10 \mu \mathrm{m}$. Additional inset magnification, $\times 2$.

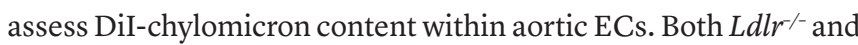
WT mice exhibited a significant increase in plasma TG levels 15 minutes after the administration of DiI-chylomicrons (Supplemental Figure 6A). Confocal microscopy analysis revealed no sig- nificant difference in DiI-chylomicron content within aortic ECs of WT and LDLR-deficient mice (Supplemental Figure 6B), suggesting that EC chylomicron uptake is independent of LDLR. We then exposed MECs in culture to DiI-chylomicrons in the absence 
or presence of apoB18, a peptide comprising the $18 \% \mathrm{NH}_{2}$-terminal sequence of apoB100 (51) that lacks the LDLR-binding site. Coincubation with apoB18 resulted in dose-dependent inhibition of DiI-chylomicron uptake (Figure 4B), suggesting that the $\mathrm{NH}_{2}$-terminus of apoB is involved in the process.

A recent genome-wide iRNA screen revealed that activin receptor-like kinase 1 (ALK1) mediates LDL uptake and transcytosis in ECs (52). Downregulation of ALK1 in cultured ECs inhibited uptake of apoB100-carrying lipoproteins LDL and VLDL, but not of apoA-I-rich HDL. Competition with LDLR did not inhibit the binding of ALK1 to LDL, suggesting that LDL-ALK1 interaction was not via the LDLR-binding region of apoB (52). However, downregulation of ALK1 with siRNA had no effect on DiI-chylomicron uptake (Figure 4C).

Knockdown of SR-BI inhibits chylomicron uptake in ECs. SR-BI is a type 1 integral membrane protein that can interact with a variety of lipoproteins via its extracellular loop $(53,54)$. SR-BI is abundantly expressed in most ECs, and it plays a central role in the metabolism and endothelial transcytosis of its canonical ligand HDL $(55,56)$. Recent studies have demonstrated that SR-BI also mediates EC uptake and transcytosis of LDL in a process that is inhibited by competition for the receptor with HDL $(57,58)$. Our competition studies showed that uptake of both DiIchylomicrons and DiI-LDL by MECs was significantly inhibited by coincubation with unlabeled HDL (Figure 4D and Supplemental Figure 7A), while unlabeled LDL or VLDL had no effect on DiIchylomicron uptake (Supplemental Figure 7, B and C). Together, these observations suggested the involvement of a receptor for HDL. We next treated MECs with antisense oligonucleotides (ASOs), which inhibited SR-BI expression in cultured MECs (Supplemental Figure 8A). The SR-BI ASOs significantly inhibited the uptake of DiI-labeled chylomicrons by MECs (Figure 4E).

We then assessed whether inhibiting SR-BI in vivo affected chylomicron uptake in aortic ECs. WT mice injected with SR-BI ASO (100 mg/kg, once a week for 3 weeks) exhibited strong systemic downregulation of SR-BI (Figure 5A), which was apparent in both aorta (Figure 5B) and aortic ECs immunostained for SR-BI (green; Figure 5, C and D). Despite the high level of similarity between SR-BI and CD36, this ASO did not reduce CD36 expression (Supplemental Figure 8B). Following SR-BI knockdown, DiI-labeled chylomicrons were administered retroorbitally and their uptake into aortic ECs assessed by confocal microscopy imaging of aortas harvested 15 minutes after injection. SR-BI knockdown induced fasting hypertriglyceridemia (Figure 5E). Circulating TG levels increased further in SR-BI-deficient mice and also increased in control mice 15 minutes after DiI-chylomicron administration (Figure 5F). SR-BI inhibition significantly blocked DiI-chylomicron uptake by aortic ECs in vivo, as compared with that in mice treated with control ASO (Figure 5G).

Downregulation of SR-BI in iLpl-- mice reduces $L D$ accumulation in aortic ECs. Our initial observations in $\mathrm{i} L \mathrm{pl}^{-/-}$mice strongly suggested that postprandial LD biogenesis in aortic ECs is not strictly dependent on LpL-mediated hydrolysis of TRLs. To determine whether SR-BI-mediated uptake of chylomicrons was a source of lipids for this process, we knocked down SR-BI in i $\mathrm{ppl}^{\mathrm{F}^{-}}$mice (Figure $5 \mathrm{H}$ ) before administration of an oral olive oil gavage. $\mathrm{i} L \mathrm{pl}^{-/-}$mice treated with control ASO or SR-BI ASO exhibited sim- ilarly elevated TG levels (Figure 5I). As expected, EC LDs were markedly increased in aortic ECs from $\mathrm{i} L \mathrm{pl}^{-/}$mice (Figure 5, J and K) 180 minutes after gavage, as compared with floxed controls. Knockdown of SR-BI in an LpL-knockout context reduced $L D$ accumulation in aortic ECs to a level similar to that of $L p l^{\mathrm{t} / \mathrm{fl}}$ controls (Figure 5, J and K).

Together, our data support a model whereby chylomicrons are internalized by ECs via SR-BI and are subsequently hydrolyzed within lysosomes. The products of intracellular chylomicron degradation fuel LD formation in ECs.

SR-BI-mediated endothelial chylomicron uptake and metabolism trigger LD formation in cocultured primary peritoneal macrophages. Patients with familial LpL deficiency exhibit impaired TG lipolysis and fasting hyperchylomicronemia, which can lead to the appearance of eruptive xanthomas (18). A 1970 study provided evidence for the chylomicron origin of lipids within macrophages of diabetic eruptive xanthomas (19). However, the authors did not observe chylomicrons within ECs nor did they find a disruption of the EC barrier. We postulated that visualization of chylomicrons within ECs would be difficult due to their rapid movement and metabolism. Our results in cultured MECs have shown that intracellular chylomicron degradation occurs rapidly, as monitored by loss of cytoplasmic apoB puncta upon removal of chylomicron-containing media (Figure 3, A and B, and Supplemental Figure 3A). In WT mice, chylomicrons were visible within ECs of aortas harvested 15 minutes after DiI-chylomicron intravenous administration. Consistent with our in vitro results, DiI-chylomicron signal declined rapidly when newly harvested aortas were maintained in FBS-free medium for 2 to 30 minutes before fixing; an approximately $50 \%$ reduction occurred within the first 2 minutes (Supplemental Figure 3B).

We hypothesized that SR-BI-mediated EC chylomicron uptake and catabolism could represent a pathway for the delivery of chylomicron-derived lipids independent of LpL activity. The bell-shaped distribution of LD occurrence in aortic ECs is consistent with a dynamic process of LD biogenesis and catabolism in response to postprandial plasma TG levels (Figure 1, A-F). LD hydrolysis in cultured ECs has been reported to result in the release of FAs into the medium, which can be internalized and reesterified by cocultured skeletal muscle cells (20). As a first step to address this hypothesis, we assessed LD formation in primary peritoneal macrophages (PMACs) cocultured with MECs. Figure 6A shows a graphic depiction of the experimental design. PMACs cocultured with MECs exposed to a chylomicron pulse contained significantly more LDs than those cocultured with untreated MECs (Figure 6B, quantification in Figure 6C). This effect was abrogated when MECs were treated with lysosomal inhibitor BafA1 following the chylomicron pulse, but not by inhibition of ATGL in chylomicron-laden MECs. Importantly, SR-BI-deficient MECs exposed to chylomicrons did not increase LD content in cocultured PMACs. These results suggest that endothelial chylomicron uptake and hydrolysis result in the release of metabolically active factors capable of triggering LD biogenesis in underlying cells.

To our surprise, our colorimetric assays were unable to detect an increase of FAs in the media of MECs exposed to a chylomicron pulse. Ultra-performance liquid chromatography coupled with quadrupole time-of-flight mass spectrometry (UPLC-TOF- 
A
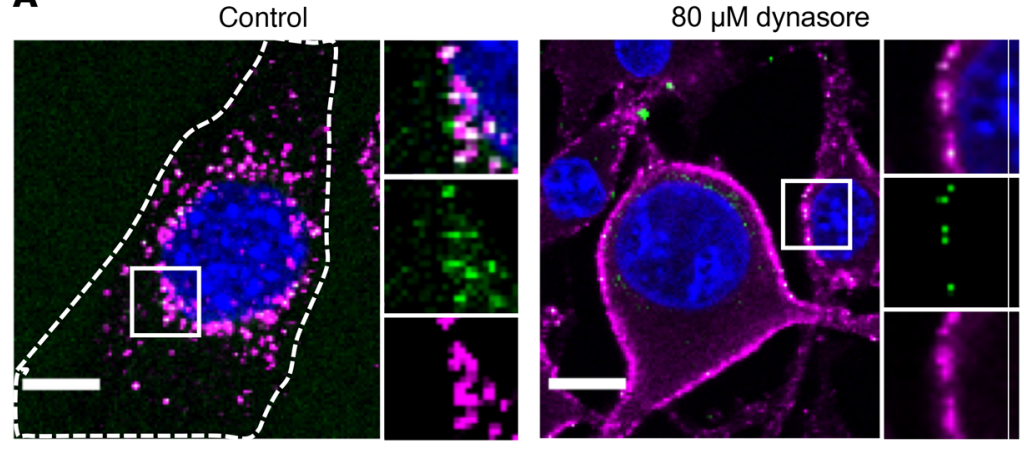

B

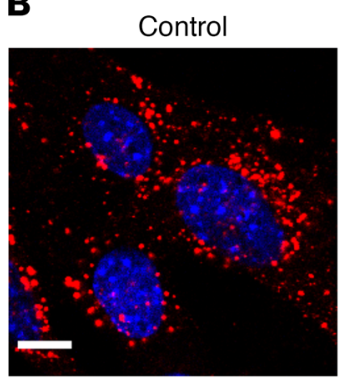

$+\operatorname{ApoB} 18(0.4 \mathrm{mg} / \mathrm{dL})$
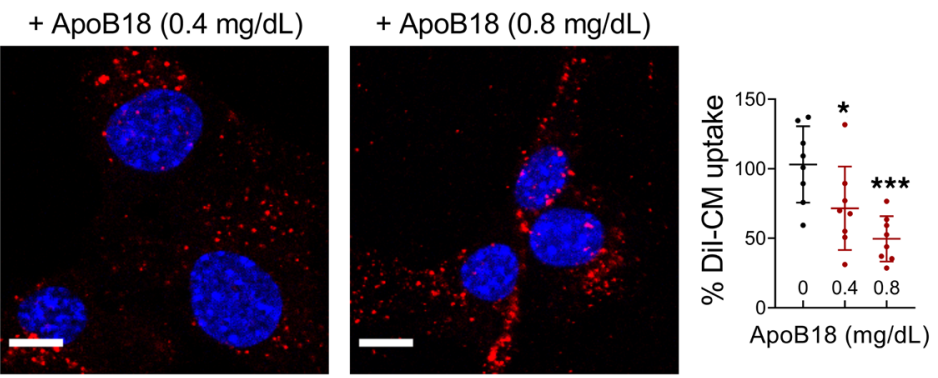

C
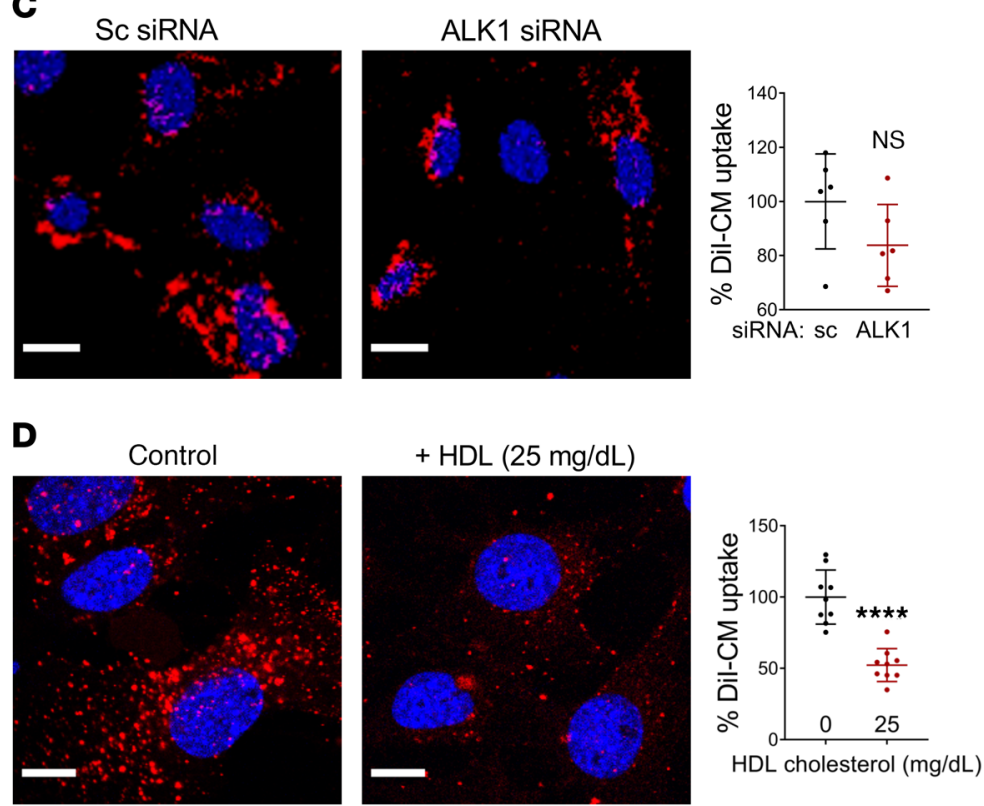

E

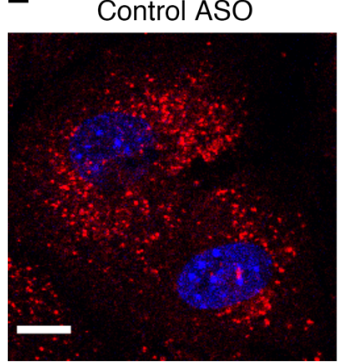

SR-BI ASO

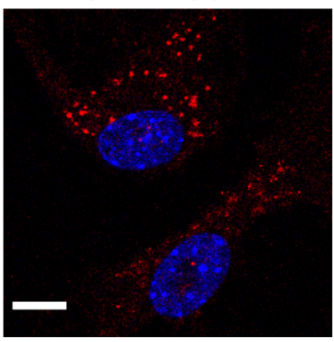

Figure 4. Mechanisms of endothelial chylomicron uptake. (A) MEC were incubated for 30 minutes with human chylomicrons in the absence (left panel) or presence of the endocytosis inhibitor dynasore (80 $\mu \mathrm{M}$, right panel). Treatment with dynasore blocked chylomicron uptake, resulting in the accumulation of apoB/BODIPY 493/503positive chylomicrons in the plasma membrane. Dashed lines show the outline of the cell. (B-E) Cultured ECs were deprived of serum overnight, and Dil-labeled human chylomicrons (4 mg/dL TG, red) were used to monitor uptake after a 30-minute pulse. (B) Coincubation with apoB18 peptide (the $\mathrm{NH}_{2}-$ terminal sequence of apoB100, 0.4 or $0.8 \mathrm{mg} / \mathrm{dL}$ ) significantly inhibited Dil-chylomicron uptake in MECs.

Data are represented as mean \pm SD of 8 independent experiments. All comparisons are with control. ${ }^{*} P<$ $0.05 ;{ }^{* * *} P<0.001$, 1-way ANOVA, Dunnet's multiple comparisons test. (C) Cells treated with control siRNA or ALK1 siRNA were deprived of serum overnight and pulsed for 30 minutes with Dil-labeled chylomicrons. Dil-chylomicron uptake was not significantly reduced in ALK1-deficient cells. (D) Dilchylomicron uptake was significantly inhibited by coincubation with unlabeled HDL $(25 \mathrm{mg} / \mathrm{dL}$ cholesterol). (E) MECs were treated with either control or SR-BI ASO for 24 hours, deprived of serum overnight, and pulsed with Dil-chylomicron. Knockdown of SR-BI significantly inhibited Dil-chylomicron uptake. Data are represented as mean \pm SEM of 4-9 independent experiments. All comparisons are with control. ${ }^{*} P<0.05$; ${ }^{* * *} P<0.0001$, Student's $t$ test. Scale bars: $10 \mu \mathrm{m}$. Additional inset magnification, $\times 2$. 
MS) analysis confirmed that FA concentration in the conditioned media of chylomicron-pulsed MECs was variable but low (0.14 $\mathrm{mM} \pm 0.12)$ and did not differ from that of untreated MECs. Further research will be required to characterize the lipids released by ECs following intracellular chylomicron hydrolysis as well as the mechanisms regulating their uptake by macrophages. PMACs from CD36-deleted mice accumulated LDs when cocultured with chylomicron-laden MECs (Supplemental Figure 9), suggesting that CD36 is not a key transporter in this process.

Downregulation of $S R-B I$ reduces $L D$ accumulation in skin macrophages of LpL-deficient mice. Finally, to assess whether SR-BImediated endothelial chylomicron uptake is a physiologically relevant source for chylomicron-derived lipids in an LpL-deficiency context, we assessed LD content in skin macrophages from i $\mathrm{Lpl}^{-1-}$ mice treated with either control or SR-BI ASO. Confocal microscopy images of skin samples from 6 mice per group (as well as from 6 nonhypertriglyceridemic $L \mathrm{pl}^{f l / f l}$ controls) immunostained for CD68 (red) and stained with BODIPY 493/503 (green) to label LDs are shown in Figure 6, D and E. Approximately 45\% of skin macrophages from i $\mathrm{pl}^{-/-}$mice contained LDs, more than double that of floxed controls. Knockdown of SR-BI in i $\mathrm{Lpl}^{-1}$ mice partially rescued this phenotype, resulting in a significant $(P<0.0001)$ reduction of the percentage of LD-positive macrophages in LpL-deficient animals.

\section{Discussion}

The distribution of chylomicron-derived lipids into parenchymal tissues has been extensively studied and largely linked to tissue expression and activity of LpL (59). The major route of uptake of chylomicron-derived FAs requires lipolysis and dissociation of nonesterified FAs (NEFA) from the apoB-containing remnant. However, an increasing body of clinical and experimental evidence suggests the existence of alternative pathways for endothelial chylomicron-derived lipid uptake independent of LpL activity.

We show that receptor-mediated uptake and lysosomal processing of chylomicrons might contribute to lipid accumulation in subendothelial macrophages. We used LpL deficiency as a model for studying movement of chylomicrons into ECs and to illustrate how chylomicrons not metabolized in the circulation can supply lipids to skin macrophages, as occurs with eruptive xanthomas. The pathway we describe illustrates a process likely to occur in LpL-deficient sites, such as in large blood vessels lined by ECs that do not express GPIHBP1 (9) and in the setting of LpL deficiency.

Internalized chylomicrons fuel LD biogenesis in ECs. In the present study, we show that LpL hydrolysis of chylomicron TG is not required for postprandial LD formation in mouse aortic ECs. LpL-deficient mice exhibited a marked increase in aortic endothelial LD content after an olive oil gavage, when chylomicron levels were severely elevated. Our in vitro studies showed that ECs internalize intact chylomicrons and that their intracellular hydrolysis at the lysosomal compartment fuels endothelial LD biogenesis.

Chylomicrons associate with and are internalized by ECs. This process seems to require the $\mathrm{NH}_{2}$ terminus of apoB and is inhibited by coincubation with HDL. Further, we have shown that downregulation of the canonical HDL receptor SR-BI inhibits endothelial chylomicron uptake. In vivo, downregulation of SR-BI inhibits uptake of DiI-labeled chylomicrons as well as LD accumulation in aortic ECs from LpL-deficient mice. Together, these results strongly support a model of LpL-independent receptor-mediated uptake and processing of chylomicron-derived lipids by ECs.

SR-BI, expressed in most ECs, was previously reported to mediate transcytosis of HDL and LDL across the EC barrier (57, $58,60,61)$. We describe an additional role for SR-BI, to mediate chylomicron uptake. Although our studies focused on ECs, the increased postprandial TG levels in our WT mice treated with ASO (Figure 5E) might reflect effects in the liver. Others have reported increased TG levels in SR-BI knockout mice as well as delayed chylomicron TG clearance (38). These findings strongly support our model whereby SR-BI mediates uptake of unhydrolyzed chylomicrons and add to the mounting evidence that SR-BI is a multifunctional player in whole-body lipid metabolism and homeostasis.

SR-BI-mediated endothelial chylomicron uptake and catabolism represent a potential pathway for the delivery of dietary $T G$ to underlying tissues. The process of endothelial chylomicron uptake and intracellular hydrolysis described in this study may result in the release by ECs of lipid products for metabolic use in subendothelial parenchymal tissues. This could be relevant to the pathophysiology of disorders associated with LpL deficiency, where chylomicron levels are severely elevated. We have shown that media from chylomicron-laden ECs induce LD biogenesis in cocultured macrophages; this process requires endothelial chylomicron hydrolysis in lysosomes. ECs with downregulated SR-BI failed to induce LD formation in cocultured PMACs. We created a mouse model of eruptive xanthomas. LpL-deficient mice exhibited a significant $(P<0.0001)$ increase in LD-containing skin macrophages, which was partially reduced by SR-BI downregulation.

Although we have focused on TG movement to skin macrophages, we suspect EC chylomicron uptake and intracellular processing in the lysosome delivers lipids to a variety of parenchymal tissues. This pathway may explain the normal adipose stores in mice and humans with LpL deficiency (62), where some lipoatrophy from defective lipid uptake by adipocytes would be expected. Consistently, LpL-deficient patients do not develop complications of fat-soluble vitamin deficiency, which do occur with abetalipoproteinemia (63). Although it is generally believed that liver metabolism of retinoids requires uptake of CMRs, and conversion of dietary retinyl ester to retinol (64), an alternative pathway, as suggested by our studies, likely delivers vitamin A to peripheral tissues in LpL-deficient patients. Because we and others (20) show that WT mice also exhibit aortic EC LDs in the postprandial period (see floxed controls in Figure 1, A and D, and Figure $5 \mathrm{~K}$ ), we suspect that this pathway is operative in mice and humans who are not LpL deficient.

We should note that the lipid-filled skin macrophages found with hyperchylomicronemia should not be equated with the cholesteryl-ester-rich foam cells seen in atherosclerosis, as cholesterol - but not TG-rich foam cells - are the pathologic signature of atherosclerosis. Although occasional cases of atherosclerotic CVD have been described in patients with homozygous LpL deficiency, these are likely to be the exception, as most investigators view nascent lipoproteins as nonatherogenic. This contrasts with partially digested or remnants of lipoproteins, which are atherogenic and a likely reason for greater CVD risk with heterozygous LpL deficiency. 
A

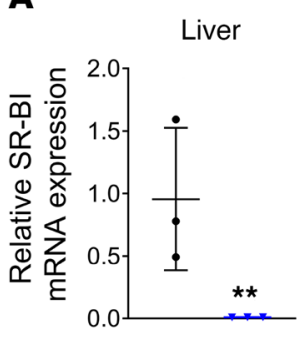

E

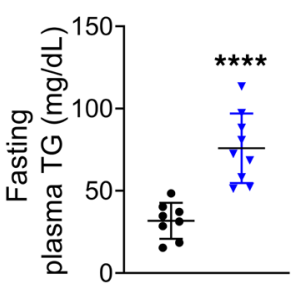

H

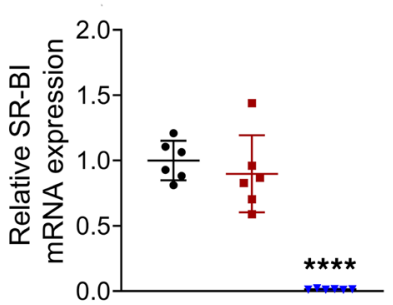

B

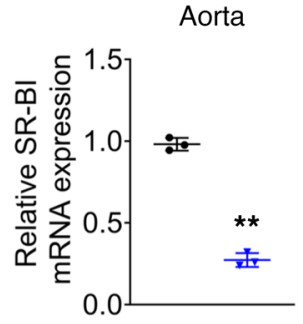

$\mathbf{F}$

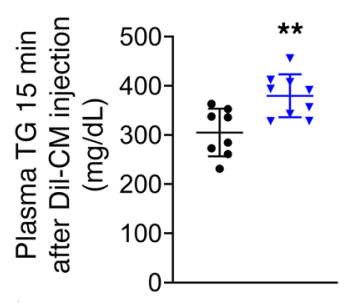

C - Control ASO

- SR-BI ASO

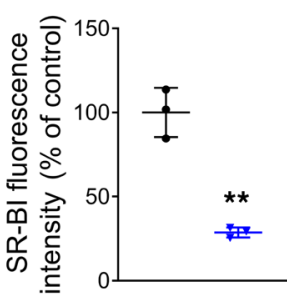

G

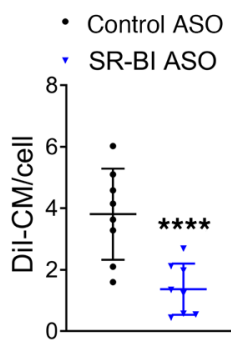

D

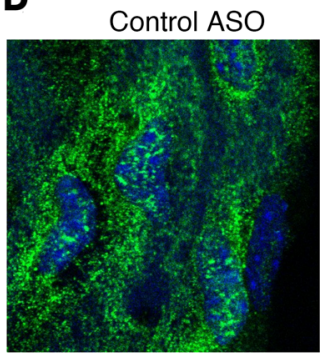

Control ASO

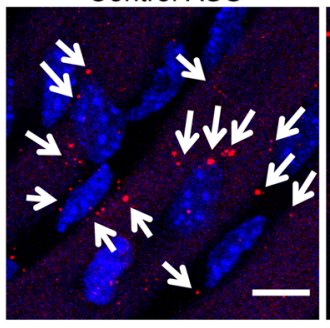

SR-BI ASO

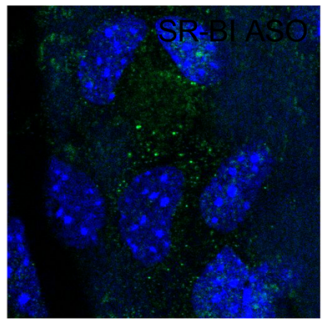

SR-BI ASO

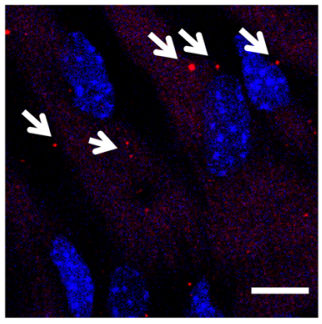

I

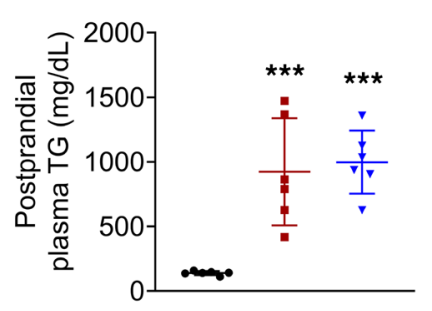

J

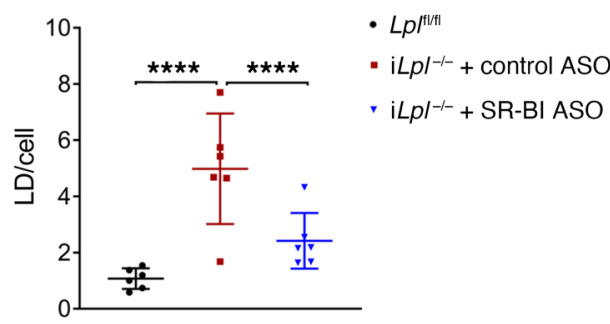

$\mathbf{K}$

$L p t^{f t / 1}$

$\mathrm{i} L p l^{-1-}+$ control ASO
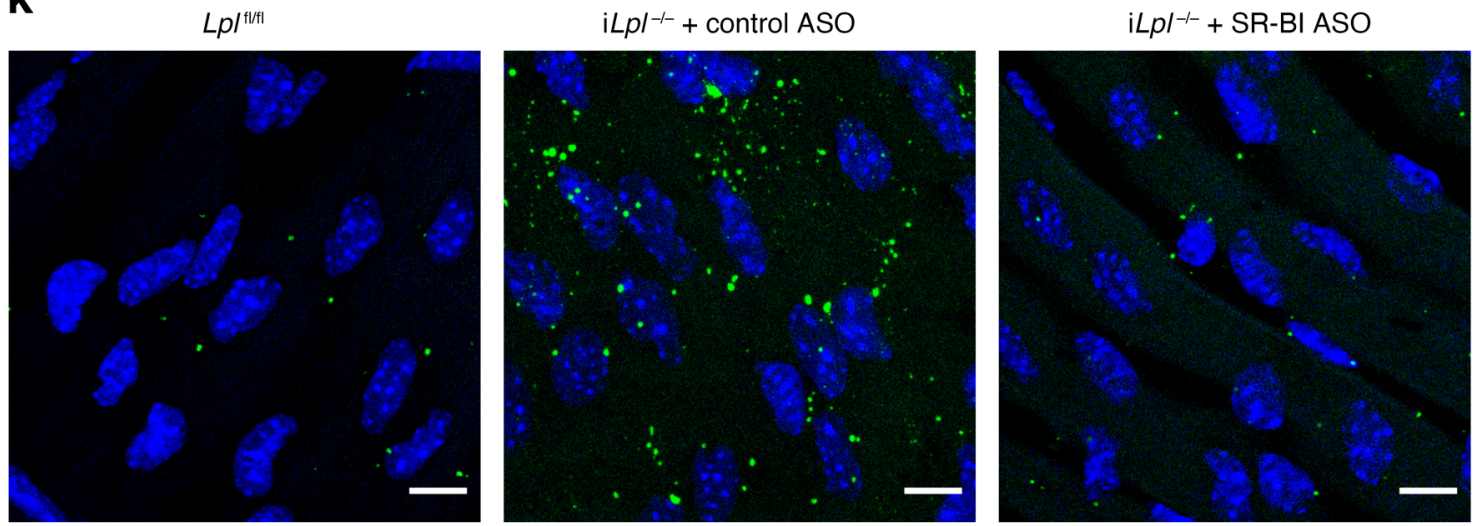

Figure 5. SR-BI deficiency inhibits in vivo aortic EC CM uptake and LD accumulation. WT, iLp|f|ffl, and $i L{ }^{1 /-}$ mice (6-8 per group) were injected with either control or SR-BI ASO (100 mg/kg) once a week for 3 weeks. Loss of SR-Bl expression was monitored by RT-PCR of liver (A) and aorta (B) as well as by immunostaining of aortic EC with SR-BI (D, green; quantified in C). ${ }^{* *} P<0.01$, Student's $t$ test. (E-C) Dil-CM $(0.5 \mathrm{mg} / \mathrm{g} \mathrm{TC})$ was administered retroorbitally to WT mice 72 hours after the last ASO injection. Mice were sacrificed and their aortas harvested 15 minutes after Dil-CM administration, and Dil-CM within aortic ECs were visualized by confocal microscope. Treatment with SR-BI ASO induced fasting hypertriglyceridemia (E), and circulating TC levels were significantly elevated 15 minutes after Dil-CM injection as compared with mice treated with control ASO (F). SR-BI knockdown significantly inhibited Dil-CM uptake in aortic ECs (F, Dil-CM shown in red). White arrows indicate Dil-CM. All comparisons are with control ASO. ${ }^{* *} P<0.01 ;{ }^{* * * * P}<0.0001$, Stu-

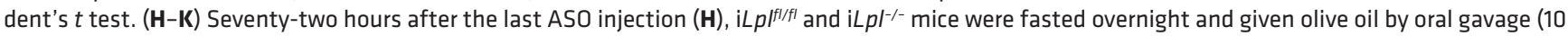
$\mathrm{ml} / \mathrm{kg}$ ). Circulating plasma TC levels (I) and aortic EC LD content (J) were assessed 180 minutes after gavage. As expected, i $\mathrm{Lp} \mathrm{F}^{-/}$mice treated with control ASO exhibited a significant increase in LD accumulation (K, middle panel) as compared with floxed controls (K, left panel). This phenotype was rescued by SR-BI downregulation (K, right panel). Data are represented as mean $\pm S D$. ${ }^{* * *} P<0.001 ;{ }^{* * *} P<0.0001$, 1-way ANOVA, Dunnet's multiple comparisons test. Scale bars: $10 \mu \mathrm{m}$. 
Aside from its enzymatic actions, LpL functions as a "bridging" molecule. By associating with cell surfaces and matrix as well as binding lipoproteins, LpL mediates internalization and matrix association of lipoprotein lipids (65-68). Very recently, Fischer et al. reported that an emulsion created from human TRLs enters brown adipose tissue ECs of cold-conditioned mice (69). This uptake requires $\mathrm{LpL}$ and $\mathrm{CD} 36$ and, as for our receptor-mediated process, leads to lysosome degradation. It is possible that in cold-acclimated mice, LpL anchoring actions are preferentially induced, possibly because the high concentrations of FAs generated inhibit LpL enzymatic activity (70). In contrast, our in vitro studies used cultured and aortic ECs lacking LpL and we found that aortic EC BODIPY + lipids were greatest in LpL-knockout mice, providing evidence for an LpL-independent pathway. In our studies, CD36 was not involved in chylomicron uptake by cultured ECs and aortic EC uptake of chylomicrons also occurred in EC-CD36 knockout. As we showed in the heart (71) and others showed in cold-induced brown adipose tissue (72), CD36 is needed for optimal uptake of lipids from VLDL but not chylomicrons $(71,73)$. Overall, aortic EC LD content appears to correlate with hyperchylomicronemia, occurs in arteries where $\mathrm{LpL}$ and its binding protein GPIHBP1 are unlikely to be found, and does not require cold exposure. Thus, tissue site, lipoprotein size or composition, physiologic conditions, and expression of LpL and CD36 differentiate these 2 pathways for TRL uptake into ECs.

In conclusion, we have defined a pathway for the uptake and metabolism of nonlipolyzed chylomicrons (Figure 7). For decades, clinicians have questioned how eruptive xanthomas, which are found in patients with severe hypertriglyceridemia, could be formed if chylomicrons were unable to cross the endothelial barrier. Our data describe a transport of chylomicrons across ECs that allows movement of lipids from circulating nascent chylomicrons into skin macrophages. This LpL-exclusive pathway likely mediates lipid delivery to parenchymal cells, allowing supply of needed lipids and fat-soluble vitamins where LpL-dependent mechanisms are less abundant.

\section{Methods}

\section{Cells}

MECs. MECs were obtained from Angiocrine Bioscience. MECs were plated in $10 \mathrm{~cm}$ tissue-culture dishes precoated with EmbryoMax 0.1\% Gelatin Solution (Sigma Aldrich). Tissue-culture medium was DMEM/Ham's F-12, supplemented with $20 \%$ FBS, $1 \%$ antibioticantimycotic, $10 \mathrm{mM}$ HEPES buffer, 1\% Glutamax, $5 \mu \mathrm{M}$ SB 431542, $50 \mu \mathrm{g} / \mathrm{mL}$ heparin, $20 \mathrm{ng} / \mathrm{mL}$ FGF, $10 \mathrm{ng} / \mathrm{mL}$ VEGF, and $50 \mu \mathrm{g} / \mathrm{mL}$ BT-203 endothelial mitogen (see Supplemental Table 1 for references). Cells were maintained in standard tissue-culture conditions $(37 \mathrm{C}$, $5 \% \mathrm{CO}_{2}$ in a humidified incubator).

Collection and culture of mouse PMACs. WT C57BL/6J mice were given an intraperitoneal injection of zymosan $(0.1 \mathrm{mg}$ in $200 \mu \mathrm{L}$ of PBS) to induce mild inflammation. At 72 hours after the injection, mice were anesthetized by isoflurane inhalation and euthanized by cervical dislocation. An incision was performed to expose the peritoneal membrane, and $5 \mathrm{~mL}$ of ice-cold PBS was injected through the membrane, followed by a gentle massage of the abdomen. Using surgical scissors, an incision was performed in the peritoneal membrane, and at least $4.5 \mathrm{~mL}$ of liquid was collected with a $1000 \mu \mathrm{L}$ pipette into a 15 $\mathrm{mL}$ conical tube. PMACs were pelleted by centrifugation ( $400 \mathrm{~g}$ for 10 minutes at $4^{\circ} \mathrm{C}$ ) and resuspended in $1 \mathrm{~mL}$ RPMI 1640 culture medium (Thermo Fisher Scientific). PMACs were plated in glass coverslips at $0.2 \times 10^{6}$ cells $/ \mathrm{mL}$ in RPMI 1640 culture medium supplemented with $10 \% \mathrm{FBS}, 1 \%$ penicillin/streptomycin (pen/strep), $10 \mu \mathrm{M}$ sodium pyruvate, $25 \mathrm{mM}$ HEPES, and $100 \mu \mathrm{M}$ amino acids. PMACs were maintained at $37^{\circ} \mathrm{C}$ and $5 \% \mathrm{CO}_{2}$ in a humidified incubator. All experiments were performed within 48 hours after PMAC collection.

Culture of HUVECs. HUVECs were cultured in M199 supplemented with EC growth supplement (Yale Vascular Biology and Therapeutics Core Facility), 10\% FBS, pen/strep (1:100), and $2.8 \mathrm{mM}$ glutamine and used up to passage 4 . For siRNA transfections, HUVECs were cultured in EGM-2 media (Lonza) with 5\% FBS.

\section{Animals}

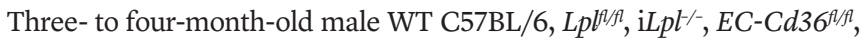
EC-Cd36-1, and $\mathrm{Ldlr}^{-/}$mice were maintained on a 12-hour light/ 12-hour dark cycle and given free access to food (laboratory chow diet) and water, except for during overnight fasting. Mice were monitored regularly to ensure their welfare.

\section{Method details}

Oral olive oil gavage and blood sampling. After a 16-hour fast, mice were given olive oil by oral gavage at $10 \mathrm{~mL} / \mathrm{kg}(\sim 300 \mu \mathrm{L})$. Blood samples were drawn at baseline and at 180 and 270 minutes after receiving the olive oil gavage. Mice were anesthetized using isoflurane, and blood was collected from the retroorbital plexus into heparinized microcapillary tubes. Blood was centrifuged at $10,000 \mathrm{~g}$ for 10 minutes for cell removal and collection of the plasma, which was then used immediately for TG measurements; the rest was frozen at $-80^{\circ} \mathrm{C}$.

Lipid measurements. Total plasma TG was measured using Infinity Triglyceride Reagent (Thermo Fisher Scientific). NEFA levels were measured using a kit (Wako Diagnostics).

ASO treatments. ASO targeting mouse SR-BI was developed and provided by Ionis Pharmaceuticals. Mice fasted for 16 hours were injected intraperitoneally with control or SR-BI ASO (GCTTCAGTCATGACTTCCTT, ISIS no. 353382) at a dose of $100 \mathrm{mg} / \mathrm{kg}$ body weigh once a week for 3 weeks. Experiments and measurements were performed 72 hours after the last ASO injection. SR-BI knockdown was assessed by real-time quantitative PCR (qRT-PCR) of samples obtained from liver (to assess systemic knockdown) and aortae (to assess local knockdown). Three thoracic aortae were pulled per sample to ensure the collection of sufficient RNA. Additionally, SR-BI knockdown in aortic ECs was assessed by en face aorta immunostaining using an SR-BI antibody (RRID; catalog AB_2182824).

Tissue gene expression. Total RNA was prepared using a GeneJET RNA Purification Kit (Thermo Scientific). One microgram of RNA was used for reverse transcription using the Verso cDNA Synthesis Kit (Thermo Scientific). qRT-PCR was performed using an ABI 7700 (Applied Biosystems). Amplification was performed using SYBR Green PCR Master Mix (Applied Biosystems). Primers used for PCR amplification were obtained from PrimerBank. Analysis was performed using Sequence Detection Software (Applied Biosciences). Data were normalized to Rn18s.

En face aorta immunostaining and neutral lipid staining. Mice were anesthetized using isoflurane and euthanized by cervical dislo- 
A
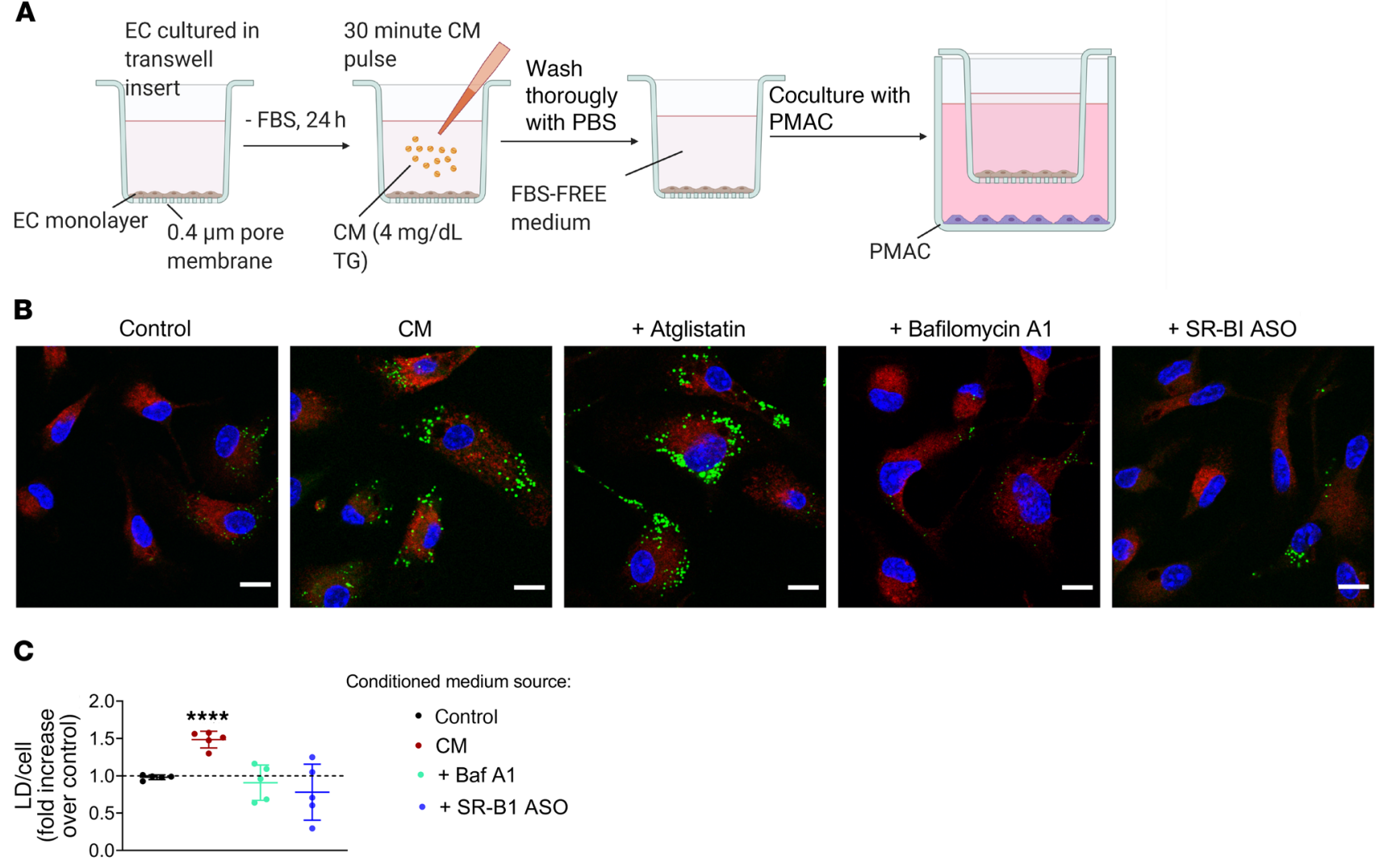

D

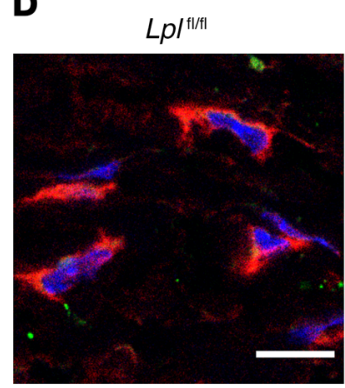

$\mathrm{i} L p l^{-/-}+$control ASO

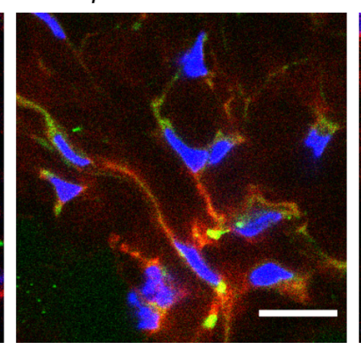

$\mathrm{i} L p l^{-/-}+\mathrm{SR}-\mathrm{Bl}$ ASO

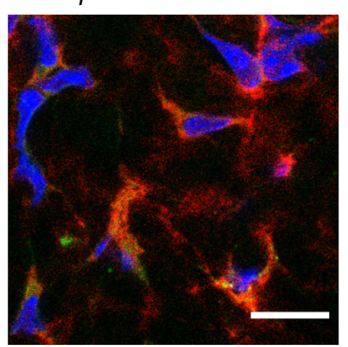

$\mathbf{E}$

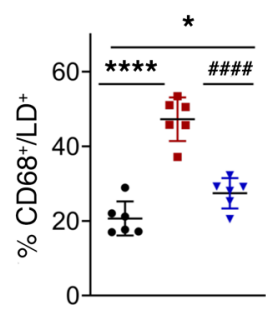

- $L p /^{\mathrm{fl} / \mathrm{fl} \mid}$

- $\mathrm{i} L p l^{-1-}+$ control ASO

- $\mathrm{i} L p l^{-1-}+\mathrm{SR}-\mathrm{BI}$ ASO

Figure 6. SR-BI deficiency reduces LD content in skin macrophages from iLpl ${ }^{-/-}$mice. (A) MECs treated with either control or SR-BI ASO were grown to confluency in Transwell inserts and deprived of FBS for 24 hours. On the day of the experiment, ECs were either left untreated (control) or exposed to a 30-minute pulse with chylomicrons, thoroughly washed, and incubated with FBS-free medium with or without atglistatin or BafA1. Inserts were then immediately placed into wells containing freshly harvested PMACs and cocultured for 4 hours. PMACs were immunostained for macrophage marker CD68 (red), and LDs were labeled with BODIPY493/503 (green). (B) Representative images of PMACs following coincubation with ECs treated as indicated. (C) LD/cell quantification. PMACs cocultured with ECs exposed to a chylomicron pulse exhibited significantly more LDs than those cocultured with untreated ECs. LD accumulation was significantly reduced in PMACs cocultured with ECs treated with BafA1 (but not atglistatin) following the chylomicron pulse. SR-BI-deficient ECs exposed to chylomicrons also failed to induce LD biogenesis in cocultured PMACs. Data are represented as mean \pm SEM of 5 independent experiments. ${ }^{* * *} P<0.0001$, 1-way ANOVA. ( $\mathbf{D}$ and $\left.\mathbf{E}\right) \mathrm{i} L \mathrm{~L}^{\mathrm{I}^{-1-}}$ mice were injected with either control or SR-BI ASO. Skin samples from the backs of 6

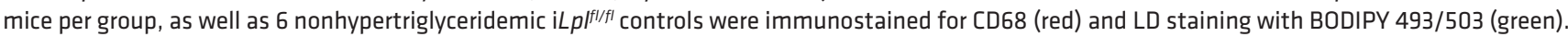
Samples were imaged by confocal microscopy (D), and the percentage of CD68/BODIPY 493/503-positive (yellow) macrophages was quantified ( $>4000$ macrophages per group) (E). LpL deficiency significantly exacerbated LD accumulation in mouse skin macrophages as compared with floxed controls. This effect was significantly reduced in i $L \mathrm{Pl}^{-/-}$mice treated with SR-BI ASO. Data are represented as mean $\pm \mathrm{SD}$. ${ }^{*} P<0.05$; ${ }^{* * *} P<0.0001$ (significantly differ-

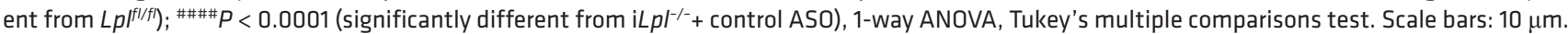

cation. Thoracic aortae were harvested in ice-cold PBS, fixed for 20 minutes in $10 \%$ formalin, and transferred to 12 -well plates containing $0.5 \mathrm{~mL}$ of a permeabilizing solution (0.1\% Triton X-100 in PBS) per well. Aortae were permeabilized for 5 minutes with rocking at room temperature (RT), then blocked using BSA (10\% in TTBS [TBS with $2.5 \%$ Tween 20]) for 30 minutes with rocking at RT. Each vessel was incubated with primary antibody (1:500 in 10\% BSA) overnight with rocking at $4^{\circ} \mathrm{C}$, followed by incubation with secondary antibody (1:1000 in 10\% BSA) for 1.5 hours with rocking at RT. LD staining with BODIPY $493 / 503$ was performed by incubation with 1:1000 by volume of a BODIPY 493/503 stock solution ( $5 \mathrm{mM}$ in DMSO) for 30 minutes at RT. Nuclear staining with DAPI was performed by incuba- 


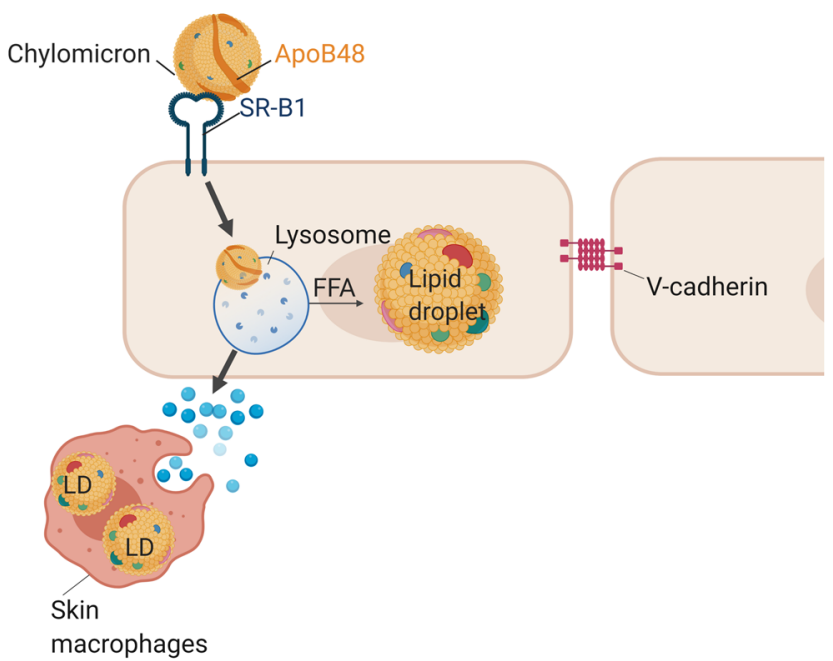

Figure 7. Model. ECs internalize unhydrolyzed chylomicrons via the SR-BI receptor. Chylomicrons are then intracellularly hydrolyzed within endothelial lysosomes, and the products of this hydrolysis fuel LD biogenesis. Additionally, intracellular chylomicron hydrolysis leads to the release by ECs of biologically active factors capable of triggering LD accumulation in underlying macrophages.

tion with 1:5000 by volume of a DAPI stock solution that contained 5 $\mathrm{mg} / \mathrm{mL}$ of DAPI in $\mathrm{H}_{2} \mathrm{O}$ ( 5 minutes at RT). Stained aortae were placed in glass coverslips with the endothelium facing down, and a drop of mounting medium was added on top. A slide glass $(22 \mathrm{~mm} \times 75 \mathrm{~mm})$ was placed on the vessel. Slides covered by 2 clean laboratory wipes and $3.5 \mathrm{~kg}$ of weight were gently placed above the slides for a maximum of 5 minutes to flatten the en face aorta sample.

In vivo aortic endothelial uptake of DiI-labeled chylomicrons. Mice were fasted overnight, and DiI-chylomicrons ( $0.5 \mathrm{mg} \mathrm{TG} / \mathrm{g}$ body weight) were injected retroorbitally. Mice were euthanized 15 minutes after injection, and thoracic aortas and blood samples were collected for DiI-chylomicron uptake and plasma TG measurements. Aortas were fixed with $10 \%$ formalin, washed 3 times with PBS, and stained with DAPI to highlight nuclei. Laser-scanning confocal microscopy (Leica SP8) was used to observe the Dil-chylomicrons within aortic ECs. When indicated, newly harvested aortas were maintained in FBS-free DMEM for 2 to 30 minutes before fixing to assess endothelial DiI-chylomicron signal decline over time.

Macrophage culture with EC-conditioned media. MECs treated with either control or SR-BI ASO were cultured in $0.4 \mu \mathrm{m}$ membrane Transwell inserts and allowed to reach confluency. Before the experiment, MECs were switched to FBS-free medium overnight to ensure minimal LD content. On the day of the experiment, MECs were either left untreated (control) or exposed to a 30-minute pulse with purified chylomicrons ( $4 \mathrm{mg} / \mathrm{dL}$ chylomicron TG) in FBS-free medium. Following the chylomicron pulse, cells were thoroughly washed with PBS and switched to FBS-free medium with or without TG hydrolysis inhibitors atglistatin and BafA1. Inserts were then immediately placed into 12 -well plates containing PMACs cultured on glass coverslips and cocultured for 4 hours. PMACs were then fixed and immunostained for the macrophage marker CD68, and LDs were stained with BODIPY 493/503. Samples were imaged by confocal microscopy.
Mouse skin macrophage sampling and immunostaining. i $\mathrm{Lpl}^{-/-}$mice were injected intraperitoneally with control or SR-BI ASO at a dose of $100 \mathrm{mg} / \mathrm{kg}$ body weight once a week for 3 weeks. At 72 hours after the last injection, mice (including $L \mathrm{pl}^{l / f l}$ controls) were gavaged with olive oil. At 180 minutes after gavage, mice were anesthetized with isofluorane and euthanized by cervical dislocation. Hair from the back was removed to expose the skin, and a $1 \times 1 \mathrm{~cm}$ region was excised using a sterile blade. Skin samples were immediately overlaid with OCT and frozen in liquid nitrogen to preserve sample integrity. OCT blocks were subsequently sectioned 3 times at NYU Langone's Pathology Core Facility. Sectioned samples were placed in slides (3 per slide) and stored at $-80^{\circ} \mathrm{C}$ for further analysis. Before immunostaining, samples were placed at RT for 5 minutes, then fixed with $10 \%$ formalin for 20 minutes at RT. Samples were then permeabilized with $0.5 \%$ Triton-X 100 (5 minutes in ice) and blocked with 3\% BSA (30 minutes at RT). Incubation with CD68 primary antibody (1:300 in 3\% BSA) and Alexa Fluor secondary antibody (1:400 in 3\% BSA) was performed by overlaying each skin section with approximately $75 \mu \mathrm{L}$ of the antibody mix, then incubating in the dark for 1 hour at RT. Following incubation with antibodies, samples were thoroughly washed with PBS. Finally, each skin section was overlaid with approximately $75 \mu \mathrm{L}$ BODIPY 493/503 (1:1000 BODIPY 493/503 stock solution in PBS) to stain LDs, and nuclei were highlighted with DAPI (1:10000 DAPI stock solution in PBS). A drop of Fluoromount Aqueous Mounting Medium (MilliporeSigma) was added to the center of the slide, and a slide glass $(22 \mathrm{~mm} \times 75 \mathrm{~mm})$ was placed on the skin sections. Skin macrophages were imaged by laser scanning confocal microscopy (Leica SP8) with an HCX PL APO lambda blue $63 \times / 1.40$ oil objective lens at RT. LDcontaining macrophages were assessed by colocalization of CD 68 and BODIPY 493/503 signals.

Lipoprotein DiI labeling and isolation. Lipoproteins from human or $\mathrm{L} L \mathrm{pl}^{-/-}$mouse plasma were isolated by differential density centrifugation. For chylomicron isolation, plasma in a Beckman Coulter tube (catalog 344059) was overlaid with an equal volume of $1.006 \mathrm{~g} / \mathrm{cm}^{3}$ density solution and centrifuged at $26,000 \mathrm{~g}$ in a TH-641 swinging bucket rotor (Thermo Scientific) for 45 minutes at $12^{\circ} \mathrm{C}$. The chylomicron layer at the top was transferred into an autoclaved $1.5 \mathrm{~mL}$ microcentrifuge tube using an autoclaved 9-inch Pasteur pipette.

For VLDL isolation, Beckman Coulter tubes containing chylomicron-free plasma and $1.006 \mathrm{~g} / \mathrm{cm}^{3}$ density solution were returned to the centrifuge and spun at $26,000 \mathrm{~g}$ for 22 hours at $12^{\circ} \mathrm{C}$. The VLDL layer at the top was transferred into an autoclaved $1.5 \mathrm{~mL}$ microcentrifuge tube using an autoclaved 9-inch Pasteur pipette. Following VLDL isolation, the rest of the $1.006 \mathrm{~g} / \mathrm{cm}^{3}$ density solution was aspirated and the remaining plasma overlaid with an equal volume of $1.12 \mathrm{~g} /$ $\mathrm{cm}^{3}$ density solution for LDL isolation. The mix was centrifuged at $26,000 \mathrm{~g}$ for 22 hours at $12^{\circ} \mathrm{C}$. The LDL layer at the top was transferred into an autoclaved $1.5 \mathrm{~mL}$ microcentrifuge tube using an autoclaved 9-inch Pasteur pipette. The remaining top $1.12 \mathrm{~g} / \mathrm{cm}^{3}$ density solution was aspirated, and the sample was overlaid by an equal volume of 1.34 $\mathrm{g} / \mathrm{cm}^{3}$ density solution for HDL isolation. The mix was centrifuged at $26,000 \mathrm{~g}$ for 46 hours at $12^{\circ} \mathrm{C}$. The HDL layer at the top was transferred into an autoclaved $1.5 \mathrm{~mL}$ microcentrifuge tube using an autoclaved 9-inch Pasteur pipette.

When required, lipoprotein labeling with DiI was performed as described by Beigneux et al.(74). Briefly, DiI was dissolved in DMSO $(3 \mathrm{mg} / \mathrm{ml})$ and $10 \mu \mathrm{l}$ of this solution was added per $250 \mu \mathrm{l}$ of human 
serum. This mixture was then incubated at $37^{\circ} \mathrm{C}$ overnight prior to lipoprotein isolation. The DiI-labeled chylomicrons were harvested and dialyzed against PBS containing 5.0 $\mu \mathrm{M}$ EDTA.

Treatment with chylomicrons in culture. Cells were seeded in glass coverslips precoated with $0.1 \%$ gelatin and allowed to reach $70 \%$ confluency. Before each experiment, cells were deprived of FBS overnight to ensure minimal LD occurrence, and all experiments were carried out in FBS-free medium. Chylomicrons were added at a concentration of $4 \mathrm{mg} / \mathrm{dL}$ TG in FBS-free medium. When indicated, cells were pretreated with $\mathrm{LpL}(10 \mathrm{U} / \mathrm{mL})$ for 1 hour at $37^{\circ} \mathrm{C}$, then washed 3 times with PBS to remove unbound LpL before incubation with chylomicrons. For pulse-chase studies, cells were exposed to chylomicrons for 30 minutes, washed thoroughly with PBS to remove uninternalized chylomicrons, and switched to FBS-free medium for 120 minutes. For competition studies, DiI-labeled chylomicrons were administered in FBS-free medium in the absence or presence of unlabeled VLDL, LDL, HDL, or apoB18 at the indicated concentrations. Endocytosis inhibitor dynasore $(80 \mu \mathrm{M})$, ATGL inhibitor atglistatin $(5 \mu \mathrm{M})$, and lysosomal proton pump inhibitor BafA1 $(100 \mathrm{nM})$ were administered when required.

In vitro delivery of SR-BI ASO. Mouse heart MECs were seeded in $0.1 \%$ gelatin-precoated 24 -well plates (in glass coverslips, for downstream imaging studies), or 6-well plates (for Western blot and qRTPCR assessment of SR-BI knockdown) and allowed to reach $40 \%$ confluency. The volumes below are for each well of a 6-well plate and could be scaled up or down according to experimental need. Before transfection with ASO, culture medium was removed and cells were overlaid with $1.8 \mathrm{~mL}$ fresh, prewarmed culture medium with FBS. Then $5 \mu \mathrm{l}$ of a $10 \mu \mathrm{M}$ ASO stock solution was added to $95 \mu \mathrm{l}$ of FBSfree DMEM in a sterile $1.5 \mathrm{ml}$ Eppendorf tube, and $4 \mu \mathrm{l}$ DharmaFECT 4 was added to $96 \mu \mathrm{l}$ of DMEM in a separate sterile $1.5 \mathrm{ml}$ Eppendorf tube. Both mixtures were combined by adding the DharmaFECT 4 mixture to the tube containing the ASO, and these were gently mixed by tapping the side of the tube. The transfection mixture was incubated at RT for 20 minutes. Cells were removed from the incubator, and $200 \mu \mathrm{l}$ of the ASO/DharmaFECT 4 mix was added to each well of the 6-well plate. The mixture was delivered dropwise and evenly throughout each well. Cells were allowed to grow for 24 hours, then switched to FBS-free medium overnight. Knockdown of SR-BI was assessed by qRT-PCR and Western blot.

Immunocytochemistry and neutral lipid staining. Following treatment, cells in glass coverslips were washed 3 times with PBS, fixed with $10 \%$ formalin (20 minutes at RT), permeabilized with $0.5 \%$ Triton-X 100 (5 minutes in ice), and blocked with 3\% BSA (30 minutes at RT). Incubation with primary antibodies (1:300 in $3 \%$ BSA) was performed overnight at $4^{\circ} \mathrm{C}$ in a humid chamber. Incubation with Alexa Fluor secondary antibodies (1:400 in 3\% BSA) was performed in the dark at RT for 45 minutes in a humid chamber. Immunostained coverslips were thoroughly washed with PBS, and neutral lipids were stained with BODIPY 493/503 (1:1000 in PBS, 30 minutes at RT).

Transwell coculture studies. MECs treated with either control or SR-BI ASO were cultured in $0.4 \mu \mathrm{m}$ pore Corning Transwell polyester membrane cell culture inserts (MilliporeSigma) for 3 days until they reached confluency, then deprived of FBS for 24 hours. On the day of the experiment, ECs were either left untreated (control) or exposed to a 30-minute pulse with chylomicrons $(4 \mathrm{mg} / \mathrm{dL}$ TG in FBS-free medi- um), after which, they were thoroughly washed and incubated with FBS-free medium with or without atglistatin $(5 \mu \mathrm{M})$ or BafA1 $(100$ $\mathrm{nM})$. Inserts were then immediately placed into 12-well plates containing freshly harvested PMACs in glass coverslips and cocultured for 4 hours. PMACs were fixed and immunostained for macrophage marker CD68. LDs were labeled with BODIPY493/503.

UPLC-TOF-MS quantification of FA in conditioned media. MECs were maintained in FBS-free media or pulsed with human chylomicrons ( $4 \mathrm{mg} / \mathrm{dL} \mathrm{TG}, 5$ replicates per condition) for 30 minutes, then thoroughly washed with PBS and switched to FBS-free medium for 4 hours. Methanol spiked with deuterated lipid standards (Avanti Lipids) in a $100 \mu \mathrm{L}$ per $50 \mathrm{~mL}$ concentration was added to conditioned media in equal parts. Lipids were extracted using $2 \times$ chloroform per sample and vortexed for 2 minutes. Samples were then centrifugated at $5635 \mathrm{~g}$ to separate a top polar layer from a bottom nonpolar layer. The bottom layer was vacufuged at $30^{\circ} \mathrm{C}$ to remove chloroform. The pelleted dry bottom layer was resuspended in $10 \mu \mathrm{L}$ of a 4:3:1 ratio of isopropanol, acetonitrile, and water. Both the top and the bottom layers were run in UPLC-TOF-MS with electrospray ionization.

Mobile phase of liquid chromatography was a binary phase consisting of A phase and B phase. A phase consisted of a 5:1:4 ratio of isopropanol, methanol, and water with a 5 millimolar ammonium acetate and 0.1\% acetic acid buffer. B phase consisted of a 99:1 ratio of isopropanol and water with a 5 millimolar ammonium acetate and a $0.1 \%$ acetic acid buffer. The column used for separation was a Zorbax Eclipse Plus C18 column with dimensions $4.6 \mathrm{~mm} \times 50 \mathrm{~mm}$ and 3.5 micron beads (Agilent). Electrospray temperatures were $300^{\circ} \mathrm{C}$, with a flow rate of $13 \mathrm{~L} / \mathrm{min}$ of nitrogen gas running in a positive or negative charge. A free FA (FFA) standard ladder was created for palmitate, oleate, and linoleate (Sigma-Aldrich). All lipids were dissolved in ethanol. FFA standards were calculated and formulated for a starting concentration of $100 \mathrm{ng} / \mu \mathrm{L}$ and serially diluted $10 \times$ to a final concentration of $0.01 \mathrm{ng} / \mu \mathrm{L}$. Standard ladder was processed and run at the same time of sample processing.

Image acquisition and analysis. All imaging acquisition was performed at NYU Langone's Microscopy Core. Samples were imaged by laser scanning confocal microscopy (Leica SP8) in the sequential scan mode with an HCX PL APO lambda blue $63 \times / 1.40$ oil objective lens at RT. BODIPY $493 / 503$ particle quantification and size analysis were performed using Image (NIH).

\section{Statistics}

Statistical analysis was performed using GraphPad Prism 7 software. Data are expressed as mean \pm SD unless otherwise indicated. Measurements between 2 groups were performed with an unpaired 2-tailed Student's $t$ test. Groups of 3 or more were analyzed by 1-way ANOVA with Dunnett's or Tukey's post hoc test as indicated. A $P$ value of less than 0.05 was considered significant. Statistical parameters for each experiment can be found within the corresponding figure legends.

\section{Study approval}

All mouse procedures were conducted in conformity with the NIH Public Health Service Policy on Humane Care and Use of Laboratory Animals and the National Research Council of the National Academy of Sciences' Guide for the Care and Use of Laboratory Animals, and were approved by the New York University Langone Medical Center Institutional Animal Care and Use Committee. 


\section{Author contributions}

IJG, NAA, AGC, and ST conceived the project. IJG, AGC, ST, WCS, SL, and JAO designed the experiments. AGC, ST, and SL conducted experiments. AGC analyzed the data. AGC and IJG wrote the original draft of the manuscript. IJG, MMH, WCS, NAA, AGC, ST, and JAO reviewed and edited the manuscript. AEM, MMH, WCS, and JAO provided resources. IJG acquired funding. IJG supervised the project. AGC and ST share the first author position. AGC is listed first because she performed the data analysis and wrote the manuscript.

\section{Acknowledgments}

Lesley-Ann Huggins fractioned the lipoproteins. Julie de Bermont and Dimitrios Nasias isolated the PMACs from WT mice. Michael Verano measured FA in media by UPLC-TOF-MS. Mouse skin samples were sectioned at the NYU Experimental Pathology Research Laboratory (partially funded by NYUCI Center support grant NIH/NCI 5 P30CA16087). All confocal microscopy experiments were carried out at NYU Langone's Microscopy Laboratory (partially supported by Cancer Center support grant P30CA016087 at the Laura and Isaac Perlmutter Cancer Center, New York, New York, USA). This study was funded by grants HL45095, 73029, and 15328 from the NIH National Heart, Lung, and Blood Institute. We thank William S. Blaner for proofreading the manuscript and providing helpful comments. Also, we appreciate insights provided by J. Witztum.

Address correspondence to: Ira J Goldberg, NYU School of Medicine, New Science Building 617, 435 East 30th Street, New York, New York 10016, USA. Email: Ira.Goldberg@nyumc.org.
1. Goldberg IJ, Bornfeldt KE. Lipids and the endothelium: bidirectional interactions. Curr Atheroscler Rep. 2013;15(11):365.

2. Zhang X, et al. Endothelial transcytosis of lipoproteins in atherosclerosis. Front Cardiovasc Med. 2018;5:130.

3. Goldberg IJ, et al. Regulation of fatty acid uptake into tissues: lipoprotein lipase- and CD36-mediated pathways. J Lipid Res. 2009;50 Suppl(Suppl):S86-S90.

4. Son NH, et al. Endothelial cell CD36 optimizes tissue fatty acid uptake. J Clin Invest. 2018;128(10):4329-4342.

5. Bastie CC, et al. CD36 in myocytes channels fatty acids to a lipase-accessible triglyceride pool that is related to cell lipid and insulin responsiveness. Diabetes. 2004;53(9):2209-2216.

6. Abumrad NA, et al. Cloning of a rat adipocyte membrane protein implicated in binding or transport of long-chain fatty acids that is induced during preadipocyte differentiation. Homology with human CD36. J Biol Chem. 1993;268(24):17665-17668.

7. Saxena U, et al. Transport of lipoprotein lipase across endothelial cells. Proc Natl Acad Sci U S A. 1991;88(6):2254-2258.

8. Mead JR, et al. Lipoprotein lipase: structure, function, regulation, and role in disease. J Mol Med (Berl). 2002;80(12):753-769.

9. Young SG, et al. GPIHBP1, an endothelial cell transporter for lipoprotein lipase. J Lipid Res. 2011;52(11):1869-1884.

10. Valdivielso P, et al. Current knowledge of hypertriglyceridemic pancreatitis. Eur J Intern Med. 2014;25(8):689-694.

11. Beigneux AP, et al. Autoantibodies against GPIHBP1 as a cause of Hypertriglyceridemia. N EnglJ Med. 2017;376(17):1647-1658.

12. Ueda M, et al. A novel APOC2 missense mutation causing apolipoprotein C-II deficiency with severe triglyceridemia and pancreatitis. JClin Endocrinol Metab. 2017;102(5):1454-1457.

13. Surendran RP, et al. Mutations in LPL, APOC2, APOA5, GPIHBP1 and LMF1 in patients with severe hypertriglyceridaemia. JIntern Med. 2012;272(2):185-196.

14. Brunzell JD, et al. Primary lipoprotein lipase deficiency. Adv Exp Med Biol. 1986;201:227-239.
15. Benlian P, et al. Premature atherosclerosis in patients with familial chylomicronemia caused by mutations in the lipoprotein lipase gene. N Engl J Med. 1996;335(12):848-854.

16. Zhang $X$, et al. Spontaneous atherosclerosis in aged lipoprotein lipase-deficient mice with severe hypertriglyceridemia on a normal chow diet. Circ Res. 2008;102(2):250-256.

17. Moutzouri E, et al. Hypocholesterolemia. Curr Vasc Pharmacol. 2011;9(2):200-212.

18. Neelamekam S, et al. The impact of lipoprotein lipase deficiency on health-related quality of life: a detailed, structured, qualitative study. Orphanet J Rare Dis. 2017;12(1):156.

19. Parker F, et al. Evidence for the chylomicron origin of lipids accumulating in diabetic eruptive xanthomas: a correlative lipid biochemical, histochemical, and electron microscopic study. JClin Invest. 1970;49(12):2172-2187.

20. Kuo A, et al. Lipid droplet biogenesis and function in the endothelium. Circ Res. 2017;120(8):1289-1297.

21. $\mathrm{He} \mathrm{C}$, et al. NanoSIMS analysis of intravascular lipolysis and lipid movement across capillaries and into cardiomyocytes. Cell Metab. 2018;27(5):1055-1066.

22. Kalluri AS, et al. Single-cell analysis of the normal mouse aorta reveals functionally distinct endothelial cell populations. Circulation. 2019;140(2):147-163.

23. Cohn JS, et al. Contribution of apoB- 48 and apoB-100 triglyceride-rich lipoproteins (TRL) to postprandial increases in the plasma concentration of TRL triglycerides and retinyl esters. J Lipid Res. 1993;34(12):2033-2040.

24. Olofsson SO, et al. Apolipoprotein B: structure, biosynthesis and role in the lipoprotein assembly process. Atherosclerosis. 1987;68(1-2):1-17.

25. Pol A, et al. Review: biogenesis of the multifunctional lipid droplet: lipids, proteins, and sites. JCell Biol. 2014;204(5):635-646.

26. Wilfling F, et al. Lipid droplet biogenesis. Curr Opin Cell Biol. 2014;29:39-45.

27. Ohsaki Y, et al. Biogenesis of cytoplasmic lipid droplets: from the lipid ester globule in the membrane to the visible structure. Biochim Biophys Acta. 2009;1791(6):399-407.

28. Robenek H, et al. Adipophilin-enriched domains in the ER membrane are sites of lipid droplet biogenesis. JCell Sci. 2006;119(pt 20):4215-4224.

29. Walther TC, Farese RV Jr. Lipid droplets and cellular lipid metabolism. Annu Rev Biochem. 2012;81:687-714.

30. Zimmermann $\mathrm{R}$, et al. Fat mobilization in adipose tissue is promoted by adipose triglyceride lipase. Science. 2004;306(5700):1383-1386.

31. Haemmerle G, et al. Defective lipolysis and altered energy metabolism in mice lacking adipose triglyceride lipase. Science. 2006;312(5774):734-737.

32. Ducharme NA, Bickel PE. Lipid droplets in lipogenesis and lipolysis. Endocrinology. 2008;149(3):942-949.

33. Bowman BJ, Bowman EJ. Mutations in subunit $C$ of the vacuolar ATPase confer resistance to bafilomycin and identify a conserved antibiotic binding site. J Biol Chem. 2002;277(6):3965-3972.

34. Moreau K, et al. Autophagosome precursor maturation requires homotypic fusion. Cell. 2011;146(2):303-317.

35. Haka AS, et al. Macrophages create an acidic extracellular hydrolytic compartment to digest aggregated lipoproteins. Mol Biol Cell. 2009;20(23):4932-4940.

36. Havel RJ. Receptor and non-receptor mediated uptake of chylomicron remnants by the liver. Atherosclerosis. 1998;141(Suppl 1):S1-S7.

37. Crawford SE, Borensztajn J. Plasma clearance and liver uptake of chylomicron remnants generated by hepatic lipase lipolysis: evidence for a lactoferrin-sensitive and apolipoprotein E-independent pathway. J Lipid Res. 1999;40(5):797-805.

38. Wiersma H, et al. Scavenger receptor BI facilitates hepatic very low density lipoprotein production in mice. J Lipid Res. 2010;51(3):544-553.

39. Ostlund-Lindqvist AM, et al. Uptake and degradation of human chylomicrons by macrophages in culture. Role of lipoprotein lipase. Arteriosclerosis. 1983;3(5):433-440.

40. Macia E, et al. Dynasore, a cell-permeable inhibitor of dynamin. Dev Cell. 2006;10(6):839-850.

41. Kirchhausen T, et al. Use of dynasore, the small molecule inhibitor of dynamin, in the regulation of endocytosis. Methods Enzymol. 2008;438:77-93.

42. Gonzales JC, et al. Apolipoproteins E and AV 
mediate lipoprotein clearance by hepatic proteoglycans. J Clin Invest. 2013;123(6):2742-2751.

43. Stanford KI, et al. Syndecan-1 is the primary heparan sulfate proteoglycan mediating hepatic clearance of triglyceride-rich lipoproteins in mice. J Clin Invest. 2009;119(11):3236-3245.

44. Yu KC, et al. LDL receptor-related protein mediates cell-surface clustering and hepatic sequestration of chylomicron remnants in LDLR-deficient mice. J Clin Invest. 2001;107(11):1387-1394.

45. Kratky D, et al. Endothelial lipase provides an alternative pathway for FFA uptake in lipoprotein lipase-deficient mouse adipose tissue. JClin Invest. 2005;115(1):161-167.

46. Fuki IV, et al. Endogenously produced endothelial lipase enhances binding and cellular processing of plasma lipoproteins via heparan sulfate proteoglycan-mediated pathway. J Biol Chem. 2003;278(36):34331-34338.

47. Goldstein JL, et al. Inhibition of proteolytic degradation of low density lipoprotein in human fibroblasts by chloroquine, concanavalin A, and Triton WR 1339. J Biol Chem. 1975;250(19):7854-7862.

48. Anzinger JJ, et al. Native low-density lipoprotein uptake by macrophage colony-stimulating factor-differentiated human macrophages is mediated by macropinocytosis and micropinocytosis. Arterioscler Thromb Vasc Biol. 2010;30(10):2022-2031.

49. Girard E, et al. The dynamin chemical inhibitor dynasore impairs cholesterol trafficking and sterol-sensitive genes transcription in human HeLa cells and macrophages. PLoS One. 2011;6(12):e29042.

50. Ritter P, et al. LDL cholesterol uptake assay using live cell imaging analysis with cell health monitoring. J Vis Exp. 2018;(141):10.3791/58564.

51. Hussain MM, et al. Apolipoprotein B binding to microsomal triglyceride transfer protein decreases with increases in length and lipidation: implications in lipoprotein biosynthesis. Biochemistry. 1997;36(42):13060-13067.

52. Kraehling JR, et al. Genome-wide RNAi screen reveals ALK1 mediates LDL uptake and transcytosis in endothelial cells. Nat Commun. 2016;7:13516.

53. Acton SL, et al. Expression cloning of SR-BI, a CD36-related class B scavenger receptor. J Biol Chem. 1994;269(33):21003-21009.

54. Kozarsky KF, et al. Overexpression of the HDL receptor SR-BI alters plasma HDL and bile cholesterol levels. Nature. 1997;387(6631):414-417.

55 . Krieger M. Scavenger receptor class B type I is a multiligand HDL receptor that influences diverse physiologic systems. J Clin Invest. 2001;108(6):793-797.

56. Shen WJ, et al. SR-BI: a unique multifunctional receptor for cholesterol influx and efflux. Annu Rev Physiol. 2018;80:95-116.

57. Armstrong SM, et al. A novel assay uncovers an unexpected role for SR-BI in LDL transcytosis. Cardiovasc Res. 2015;108(2):268-277.

58. Huang L, et al. SR-BI drives endothelial cell LDL transcytosis via DOCK4 to promote atherosclerosis. Nature. 2019;569(7757):565-569.

59. Savonen R, et al. The tissue distribution of lipoprotein lipase determines where chylomicrons bind. J Lipid Res. 2015;56(3):588-598.

60. Rohrer L, et al. High-density lipoprotein transport through aortic endothelial cells involves scavenger receptor BI and ATP-binding cassette transporter G1. Circ Res. 2009;104(10):1142-1150.

61. Vaisman BL, et al. Endothelial expression of scavenger receptor class B, type I protects against development of atherosclerosis in mice. Biomed Res Int . 2015;2015:607120.

62. Garcia-Arcos I, et al. Adipose-specific lipoprotein lipase deficiency more profoundly affects brown than white fat biology. J Biol Chem. 2013;288(20):14046-14058.

63. Zamel R, et al. Abetalipoproteinemia: two case reports and literature review. Orphanet J Rare Dis. 2008;3:19.

64. O'Byrne SM, Blaner WS. Retinol and retinyl esters: biochemistry and physiology. J Lipid Res. 2013;54(7):1731-1743.
65. Saxena U, et al. Lipoprotein lipase increases low density lipoprotein retention by subendothelial cell matrix. J Clin Invest. 1992;89(2):373-380.

66. Rumsey SC, et al. Lipoprotein lipase-mediated uptake and degradation of low density lipoproteins by fibroblasts and macrophages. J Clin Invest. 1992;90(4):1504-1512.

67. Mulder M, et al. Heparan sulphate proteoglycan are involved in the lipoprotein lipase-mediated enhancement of the cellular binding of very low density and low density lipoproteins. Biochem Biophys Res Commun. 1992;185(2):582-587.

68. Merkel M, et al. Inactive lipoprotein lipase (LPL) alone increases selective cholesterol ester uptake in vivo, whereas in the presence of active LPL it also increases triglyceride hydrolysis and whole particle lipoprotein uptake.J Biol Chem. 2002;277(9):7405-7411.

69. Fischer AW, et al. Lysosomal lipoprotein processing in endothelial cells stimulates adipose tissue thermogenic adaptation. Cell Metab. 2021;33(3):547-564.

70. Baginsky ML, Brown WV. Differential characteristics of purified hepatic triglyceride lipase and lipoprotein lipase from human postheparin plasma. J Lipid Res. 1977;18(4):423-437.

71. Bharadwaj KG, et al. Chylomicron- and VLDL-derived lipids enter the heart through different pathways: in vivo evidence for receptorand non-receptor-mediated fatty acid uptake. JBiol Chem. 2010;285(49):37976-37986.

72. Bartelt A, et al. Brown adipose tissue activity controls triglyceride clearance. Nat Med. 2011;17(2):200-205.

73. He C, et al. NanoSIMS analysis of intravascular lipolysis and lipid movement across capillaries and into cardiomyocytes. Cell Metab. 2018;27(5):1055-1066.

74. Beigneux AP, et al. Glycosylphosphatidylinositol-anchored high-density lipoprotein-binding protein 1 plays a critical role in the lipolytic processing of chylomicrons. Cell Metab. 2007;5(4):279-291 